\title{
Application of Nonredundant Sampling Representations of Electromagnetic Fields to NF-FF Transformation Techniques
}

\author{
Ovidio M. Bucci ${ }^{1}$ and Claudio Gennarelli ${ }^{2}$ \\ ${ }^{1}$ Dipartimento di Ingegneria Biomedica, Elettronica e delle Telecomunicazioni, University of Naples Federico II, \\ Via Claudio 21, 80125 Napoli, Italy \\ ${ }^{2}$ Dipartimento di Ingegneria Elettronica ed Ingegneria Informatica, University of Salerno, via Ponte Don Melillo, \\ 84084 Fisciano, Italy \\ Correspondence should be addressed to Claudio Gennarelli, gennar@diiie.unisa.it
}

Received 31 August 2011; Accepted 23 December 2011

Academic Editor: Daniël Janse van Rensburg

Copyright (๑) 2012 O. M. Bucci and C. Gennarelli. This is an open access article distributed under the Creative Commons Attribution License, which permits unrestricted use, distribution, and reproduction in any medium, provided the original work is properly cited.

\begin{abstract}
An overview of the application of the band-limitation properties and nonredundant sampling representations of electromagnetic fields to NF-FF transformations is presented. The progresses achieved by applying them to data acquired on conventional NF scanning surfaces are discussed, outlining the remarkable reduction in the number of needed NF samples and measurement time. An optimal sampling interpolation expansion for reconstructing the probe response on a rotational scanning surface from a nonredundant number of its samples is also discussed. A unified theory of the NF-FF transformations with spiral scannings, which allow a remarkable reduction of the measurement time, is then reviewed by describing a sampling representation of the voltage on a quite arbitrary rotational surface from its nonredundant samples collected on a proper spiral wrapping it. Some numerical and experimental results assessing the effectiveness of the considered NF-FF transformations are shown too.
\end{abstract}

\section{Introduction}

Sampling representations play a significant role in many topics of applied electromagnetism, such as antenna analysis, synthesis and diagnostics, antenna near-field (NF) and farfield (FF) measurements, microwave nondestructive testing and imaging, and so forth. They are usually more convenient and efficient than the representations of electromagnetic (EM) fields based on modal or asymptotic expansions. This is due to the fact that the expansion coefficients are the field values at the sampling points (i.e., the available measured or computed quantities), and the basis functions are simple and universal. Accordingly, they can be employed to represent in an efficient and accurate way the radiated (or scattered) EM field on substantially arbitrary surfaces. The possibility and effectiveness of the sampling representations rely on the quasi-band-limitedness property of the field radiated (or scattered) by arbitrary (non super directive) sources. As a matter of fact, it is shown in [1] that the fields radiated by sources enclosed in a sphere of radius $a$ and observed on an analytical curve $C$ external to it can be very well approximated by spatially band-limited functions. For large sources, the bandwidth is practically equal to $\beta a$ ( $\beta$ being the wave number), provided that the phase propagation factor is extracted from the field expression, that a normalized arc length is used to parameterize $C$ and that $C$ is some wavelength far from the source. Since a regular surface $\mathcal{M}$ can be described by two families of coordinate curves, the extension to this last case is straightforward. This property implies that the field over any regular surface surrounding the source can be accurately represented by a standard cardinal series (CS).

This possibility has been first successfully applied to the NF-FF transformation with cylindrical scanning [2]. Unlike the standard approaches $[3,4]$, the number of samples on each ring decreases as we move from the central rings to the peripheral ones, and the linear spacing between the rings also grows when the radius of the scanning cylinder increases. 
Accordingly, it can become significantly greater than halfwavelength, then commonly accepted in literature as the maximum possible sampling interval. However, the use of the CS, while completely satisfactory from the accuracy point of view, has the drawback that all samples (or, at least, all the relevant ones) must be considered in evaluating the field at a given point, otherwise a relatively large truncation error is introduced, due to the slow decay of the sampling functions (It must be noted that this slow decay becomes beneficial when we want extrapolate the measured data, f. i., to reduce the error due to the unavoidable truncation of the measurement zone [5]). Such a slow decay leads to a further difficulty related to the fact that in practice we deal with inaccurate data. Provided that we are well above the noise level, the measured results are usually affected by an approximately constant relative error, so that the absolute error corresponding to the highest field values can be relatively large. This error is spread out by the sampling functions without a severe attenuation, and this can induce significant relative error in the zones where the field level is low.

The above difficulties have been overcome by resorting to optimal sampling interpolations (OSIs) of central type $[6,7]$, in which only relatively few samples in the neighbourhood of the output point are used in the reconstruction. These algorithms minimize the truncation error for a given number of retained samples and are more stable than the CS expansions with respect to random errors affecting the data. By taking advantage of the aforementioned band-limitation property, effective NF-FF transformations with plane-polar [8] and bipolar scanning [9-12] have been developed. They make use of efficient OSI expansions to reconstruct the NF data needed by the classical plane-rectangular NF-FF transformation from the knowledge of the acquired ones. Although a significant reduction on the number of required samples is obtained in such a way, their number increases indefinitely when the cylinder height or the radius of the scanning zone approach infinity. This shortcoming is a typical companion of unbounded observation surfaces, such as the cylinder and the plane. On the other hand, for a spherical scanning geometry, the number of needed samples is finite, independent of the sphere size, and essentially coincident with the number of degrees of freedom of the field [13], that is, the number of independent parameters necessary to represent it with a given precision. As the knowledge of the field on a sphere encircling the source uniquely determines the field at its exterior, this clearly shows that above representations are redundant.

Apart from above drawback, the hypothesis that the antenna is contained in a sphere is not always the most natural one. If the source geometry departs significantly from the spherical one, this choice leads again to redundancy (see Section 2) and does not make it possible to consider observation domains close to the source.

Note that the redundancy of the representation, which in direct type problems affects the efficiency but not the stability of the algorithms, becomes of crucial importance in inverse problems (antenna synthesis, inverse scattering, phase retrieval, image restoration, etc.) where, due to illposedness, it is not possible to recover a number of inde- pendent parameters greater than the number of degrees of freedom [14].

Nonredundant sampling representations of the EM fields radiated or scattered by sources enclosed in a convex domain $\mathscr{D}$ of finite size, bounded by a surface $\Sigma$ with rotational symmetry, and observed on a regular surface $\mathcal{M}$ external to $\mathscr{D}$ and having the same symmetry have been developed in [15], by properly generalizing the approach introduced in [1]. Now, the number of required samples is finite also for an unbounded observation domain, independent of its shape and size, and essentially coincident with the number of degrees of freedom of the field. This is obtained by a proper choice of the phase factor to extract from the field and of the parameterizations adopted to describe $\mathcal{M}$. Of course, OSI expansions can be exploited for minimizing the truncation error, allowing effective sampling representations also on subdomains of $\mathcal{M}$. Moreover, since, as shown in [16], the output of a nondirective probe (the voltage, say) has the same effective spatial bandwidth of the field radiated by the source, the nonredundant sampling representations can be directly applied to it. This allows to accurately reconstruct the NF data required to carry out the corresponding NFFF transformation, starting from a nonredundant set of measurements. In this way, effective NF-FF transformations requiring a minimum number of measurements have been developed, for various source shapes, in plane-polar [1618], bipolar [19], planar wide mesh [20], cylindrical [21$23]$, and spherical $[24,25]$ scanning geometries. It must be stressed that the reduction in the number of the NF samples to be acquired reflects in a decrease of the measurement time, which is a very important issue, since nowadays such a time is much larger than that needed to carry out the NFFF transformation.

For drastically reducing the acquisition time, the use of the modulated scattering technique, employing arrays of scattering probes, which allows a very fast electronic scanning, has been proposed since 1988 [26]. However, apart from measurement precision issues, antenna testing facilities based on such a technique are not very flexible. Anyway, exploitation of nonredundant sampling theory could allow to reduce the number of required probes. Moreover, scanning schemes for reducing the NF data acquisition time in the spherical near- field measurements of electrically large antennas have been recently proposed and experimentally validated in [27-29].

Another way to reduce the measurement time is the use of spiral scanning techniques. They have been implemented, as suggested by Yaccarino et al. in [30], by means of continuous and synchronized movements of the positioning systems of the probe and antenna under test (AUT). Accurate, stable and efficient NF-FF transformations with helicoidal, planar and spherical spiral scanning have been developed in the last decade [31-41]. They rely on nonredundant sampling representations to reconstruct the NF data needed by the classical NF-FF transformation corresponding to the adopted scanning surface, by interpolating, via appropriate OSI formulas, the nonredundant samples collected on the spiral. The AUT has been assumed enclosed in the smallest sphere able to contain it in [31-34], whereas more effective AUT 
modellings, that allow a further reduction of required NF samples when dealing with antennas having one or two predominant dimensions, have been adopted in [35-41]. A unified theory for spiral scanning and nonspherical antennas is reported in [41].

However, when dealing with aperture antennas, such an additional a priori information available on the AUT can be conveniently exploited to provide the aperture field with an effective representation, suitably accounting for its shape and size as well as for its radiating features, thus further reducing the number of required samples [42-44].

As a final remark, note that sampling representations have been also employed to develop NF-FF transformation techniques from phaseless NF data in the plane-polar [45], bipolar [46, 47], or cylindrical [48] scanning geometries.

In this paper we provide an overview of the application of the nonredundant sampling representations to NF-FF transformations, with particular emphasis on spiral scanning. The paper is organized as follows. The theoretical results concerning the nonredundant sampling representation of EM fields are summarized in Section 2, highlighting the role of the optimal parameterization and phase factor. The ellipsoidal (oblate and prolate) modelling of the source, suitable for quasiplanar and elongated antennas, is explicitly reported. In Section 3, a two-dimensional OSI expansion for reconstructing the probe voltage on an arbitrary rotational scanning surface from its nonredundant samples is presented, and representative numerical and experimental results relevant to the cylindrical scanning case are reported. The unified theory of the NF-FF transformations with spiral scanning, for antennas with two dimensions very different from the third one [41], is described in Section 4, by presenting a sampling representation of the probe voltage on a quite arbitrary rotational surface from its nonredundant samples collected on a proper spiral wrapping the surface. Application of this theory to helicoidal scanning and elongated antennas is also reported in the same Section, and experimental results validating the related NF-FF transformation are shown. At last, conclusions are summarized in Section 5.

\section{Nonredundant Sampling Representations of Electromagnetic Fields}

Let us consider the field radiated by a nonsuperdirective source $S$, enclosed in a convex domain $\mathcal{D}$ of finite size, bounded by a surface $\Sigma$ with rotational symmetry, and observed on a regular surface $\mathcal{M}$, external to $\mathcal{D}$ and with the same symmetry. Since $\mathcal{M}$ can be described by two families of coordinate curves, namely meridian curves and azimuthal circumferences, in the following we deal with the field representation over a regular curve $C$ described by a regular parameterization $\underline{r}=\underline{r}(\xi)$. As shown in $[1,15]$, the "band-limitation" error, which occurs when the "reduced electric field" $\gamma(\xi)$, being a regular function of $\xi$, is approximated by a spatially band-limited function, becomes negligible as the bandwidth exceeds the critical value

$$
W_{\xi}=\max _{\xi}[w(\xi)]=\max _{\xi}\left[\max _{\underline{r}^{\prime}}\left|\frac{\mathrm{d} \gamma(\xi)}{\mathrm{d} \xi}-\beta \frac{\partial R\left(\xi, \underline{r}^{\prime}\right)}{\partial \xi}\right|\right] .
$$

In (2), $\underline{r}^{\prime}$ denotes the source point and $R=\mid \underline{r}(\xi)-$ $\underline{r}^{\prime} \mid$. In fact, for large sources and observation curves, some wavelengths far from $\mathcal{D}$, such an error exhibits a steplike behaviour, decreasing more than exponentially as the bandwidth exceeds $W_{\xi}[1,15]$. Accordingly, it can be effectively controlled by choosing a bandwidth slightly larger than $W_{\xi}$. And so, $\underline{F}(\xi)$ can be represented on $C$ by a CS in $\xi$, with a sampling rate slightly larger than $W_{\xi} / \pi$. To obtain a nonredundant representation, that is, to minimize the number of required samples, it is shown in [15] that two conditions must be satisfied; namely,

(1) $\gamma$ must be chosen in such a way that its derivative is equal to the mean between the maximum and minimum values of $\beta \partial R / \partial \xi$ when $\underline{r}^{\prime}$ varies in $\mathcal{D}$;

(2) the parameter $\xi$ must be such that the local bandwidth $w(\xi)$ is constant with $\xi$.

Condition (2) arises from the fact that if $w(\xi)$ is variable with $\xi$, the sample spacing becomes unnecessary small in the zones wherein $w(\xi)$ is smaller than its maximum value $W_{\xi}$.

According to the above conditions, by denoting with $s$, the curvilinear abscissa along the curve $C$ and assuming $\gamma(0)=0$ and $\xi(0)=0$, it results in $[15]$ :

$$
\begin{aligned}
\gamma & =\gamma(\xi)=\frac{\beta}{2} \int_{0}^{s(\xi)}\left[\max _{\underline{r}^{\prime}} \frac{\partial R}{\partial s}+\min _{\underline{r}^{\prime}} \frac{\partial R}{\partial s}\right] \mathrm{d} s \\
& =\frac{\beta}{2} \int_{0}^{s}\left[\max _{\underline{r}^{\prime}} \hat{R} \cdot \hat{t}+\min _{\underline{r}^{\prime}} \hat{R} \cdot \hat{t}\right] \mathrm{d} s, \\
\xi & =\frac{\beta}{2 W_{\xi}} \int_{0}^{s}\left[\max _{\underline{r}^{\prime}} \frac{\partial R}{\partial s}-\min _{\underline{r}^{\prime}} \frac{\partial R}{\partial s}\right] \mathrm{d} s \\
& =\frac{\beta}{2 W_{\xi}} \int_{0}^{s}\left[\max _{\underline{r}^{\prime}} \hat{R} \cdot \hat{t}-\min _{\underline{r}^{\prime}} \hat{R} \cdot \hat{t}\right] \mathrm{d} s,
\end{aligned}
$$

where $\hat{t}$ is the unit vector tangent to $C$ at the observation point $P, \widehat{R}$ is the unit vector pointing from the source point to $P$, and $(\cdot)$ denotes the inner product. According to (4), a change of $W_{\xi}$ is reflected in a simple change of scale for $\xi$.

When $C$ is a meridian curve (see Figure 1) and $\hat{t}$ is external to the cone of vertex $P$ tangent to $\Sigma$, the extreme values of $\hat{R} \cdot \hat{t}$ occur at the two tangency points $P_{1,2}$ on $C^{\prime}$ (intersection curve between the meridian plane and $\Sigma$ ). By taking into account that [15]

$$
\left.\frac{\partial R}{\partial s}\right|_{s_{1,2}^{\prime}}=\frac{\mathrm{d} R_{1,2}}{\mathrm{~d} s} \pm \frac{\mathrm{d} s_{1,2}^{\prime}}{\mathrm{d} s},
$$

$$
\underline{F}(\xi)=\underline{E}(\xi) \mathrm{e}^{\mathrm{j} \gamma(\xi)}
$$




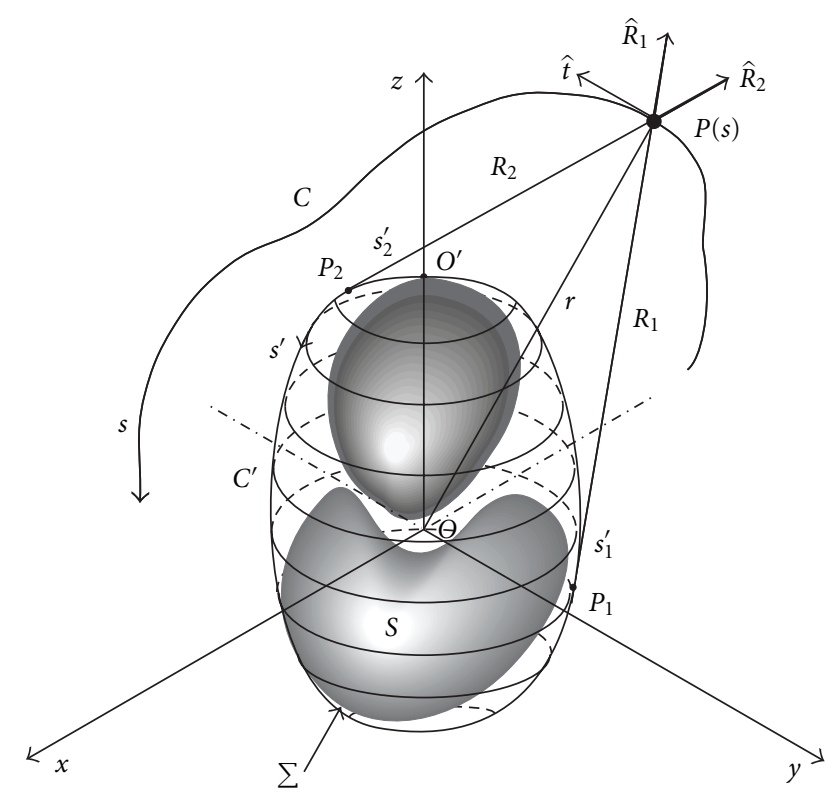

FIgUre 1: Relevant to a meridian observation curve.

where $s_{1,2}^{\prime}$ are the arc length coordinates of $P_{1,2}$ and $R_{1,2}$ the distances from $P$ to $P_{1,2}$ (see Figure 1 ), and choosing $W_{\xi}=$ $\beta \ell^{\prime} / 2 \pi$ ( $\ell^{\prime}$ being the length of $C^{\prime}$ ), we have

$$
\begin{aligned}
& \gamma=\frac{\beta}{2}\left[R_{1}+R_{2}+s_{1}^{\prime}-s_{2}^{\prime}\right], \\
& \xi=\frac{\pi}{\ell^{\prime}}\left[R_{1}-R_{2}+s_{1}^{\prime}+s_{2}^{\prime}\right] .
\end{aligned}
$$

It is useful to note that, according to (7), the angularlike parameter $\xi$ covers a $2 \pi$ range when $P$ encircles the source once. Moreover, the number of samples at Nyquist spacing $\left(\Delta \xi=\pi / W_{\xi}\right)$ on a closed meridian curve $C$ is always finite (also when this last is unbounded) and equal to $N_{\xi}=$ $2 \pi / \Delta \xi=2 \ell^{\prime} / \lambda, \lambda$ being the wavelength.

When $C$ is an azimuthal circumference of radius $\rho$, the extreme values of $\hat{R} \cdot \hat{t}$ are opposite and constant along it. It follows from (3) and (4) that $\gamma$ is constant, and any parameter proportional to the arc length is optimal. Accordingly, it is convenient to use the azimuthal angle $\varphi$ as parameter and to choose for $\gamma$ the value relevant to any meridian curve passing through the observation point on $C$. The corresponding bandwidth is [15]

$$
\begin{aligned}
W_{\varphi}= & \frac{\beta}{2} \max _{z^{\prime}}\left(R^{+}-R^{-}\right) \\
=\frac{\beta}{2} \max _{z^{\prime}} & \left(\sqrt{\left(z-z^{\prime}\right)^{2}+\left(\rho+\rho^{\prime}\left(z^{\prime}\right)\right)^{2}}\right. \\
& \left.-\sqrt{\left(z-z^{\prime}\right)^{2}+\left(\rho-\rho^{\prime}\left(z^{\prime}\right)\right)^{2}}\right),
\end{aligned}
$$

wherein $\rho^{\prime}\left(z^{\prime}\right)$ is the equation of $\Sigma$ in cylindrical coordinates, and $R^{+}, R^{-}$are the maximum and minimum distance, respectively, from each circumference describing the surface $\Sigma$ to the observation point $P$. It can be easily shown that the maximum is attained on that zone of the surface $\Sigma$ lying on the same side of the observation circumference with respect to its maximum transverse circle. When the observation circle moves toward infinity, it results in $R^{+}=R^{-}+2 \rho^{\prime} \sin \vartheta$, and, accordingly, we have

$$
W_{\varphi}=\beta \rho_{\max }^{\prime} \sin \vartheta,
$$

$\rho^{\prime}{ }_{\text {max }}$ being the maximum transverse radius of $\Sigma$ and $\vartheta$ the polar angle of the circle points. It can be shown [15] that the number of field samples on any closed observation surface (also unbounded) surrounding the source is

$$
N_{0} \cong \frac{\text { area of } \Sigma}{(\lambda / 2)^{2}}
$$

Taking into account that the two tangential components of the electric field over any surface enclosing the source uniquely determine the field outside it, we realize that the number of degrees of freedom of the field radiated by an arbitrary source within $\mathscr{D}$ is practically equal to $2 N_{0}$, as a number of samples slightly larger than $N_{0}$ ensures a negligible representation error.

According to (10), $D$ must fit the antenna geometry, as much as possible, in order to minimize the overall number of samples.

As representative examples, we report in the following the results relative to ellipsoidal geometries. A prolate ellipsoid, having major and minor semiaxes equal to $a$ and $b$ (Figure 2), is an effective modelling for elongated antennas, whereas an appropriate modelling for quasiplanar ones is obtained by choosing $\Sigma$ coincident with an oblate ellipsoid. These models are quite general and contain the spherical and planar ones as particular cases. For both types of ellipsoids, it can be shown [15] that in any meridian plane the curves $\gamma=$ const and $\xi=$ const are ellipses and hyperbolas confocal to $C^{\prime}$. Accordingly, $\xi$ and $\gamma$ are functions only of the elliptic coordinates $u=\left(r_{1}-r_{2}\right) / 2 f$ and $v=\left(r_{1}+r_{2}\right) / 2 a$, where $2 f$ is the focal distance of the ellipse $C^{\prime}$ and $r_{1,2}$ the distances from observation point $P$ on the meridian curve to the foci (Figure 2).

By straightforward but lengthy computations, from (6) and (7), it results in [15]

$$
\begin{gathered}
\gamma=\beta a\left[v \sqrt{\frac{v^{2}-1}{v^{2}-\varepsilon^{2}}}-\mathrm{E}\left(\cos ^{-1} \sqrt{\frac{1-\varepsilon^{2}}{v^{2}-\varepsilon^{2}}} \mid \varepsilon^{2}\right)\right], \\
\xi=\frac{\pi}{2} \begin{cases}\frac{\mathrm{E}\left(\sin ^{-1} u \mid \varepsilon^{2}\right)}{\mathrm{E}\left(\pi / 2 \mid \varepsilon^{2}\right)}+1 & \text { prolate ellipsoid, } \\
\frac{\mathrm{E}\left(\sin ^{-1} u \mid \varepsilon^{2}\right)}{\mathrm{E}\left(\pi / 2 \mid \varepsilon^{2}\right)} & \text { oblate ellipsoid, }\end{cases}
\end{gathered}
$$

where $\varepsilon=f / a$ is the eccentricity of $C^{\prime}$, and $\mathrm{E}(\cdot \mid \cdot)$ denotes the elliptic integral of second kind.

Relation (12) is valid when the angle 9 lies in the range [0, $\pi / 2$ ]. The case in which $\vartheta$ belongs to $[\pi / 2, \pi]$ can be easily handled by determining the value $\xi^{\prime}$ corresponding to the point specified by the angle $\pi-\vartheta$ and then putting $\xi=\pi-\xi^{\prime}$. 


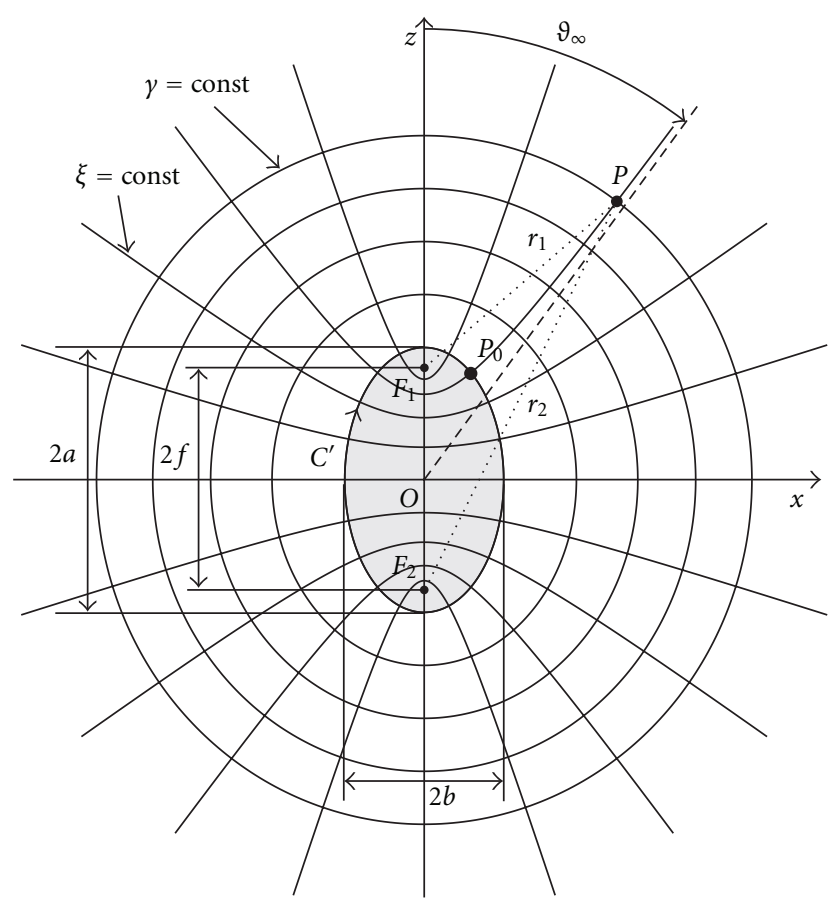

FIGURE 2: Ellipsoidal source modelling: prolate case.

With reference to an azimuthal circumference, it can be shown [15] that the same value of $W_{\varphi}$ corresponds to all transverse circles on the hyperboloid of rotation fixed by $\xi$. Accordingly, moving the circle to infinity and taking (9) into account, we get the following:

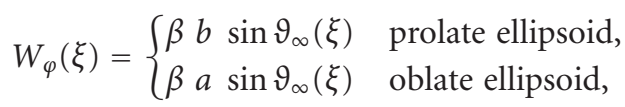

wherein $\vartheta_{\infty}$ is the polar angle of the asymptote to the hyperbola through $P$ (Figure 2 ) and is given by

$$
\vartheta_{\infty}= \begin{cases}\sin ^{-1} u+\frac{\pi}{2} & \text { prolate ellipsoid, } \\ \sin ^{-1} u & \text { oblate ellipsoid. }\end{cases}
$$

In order to show the effect of multiplying the field by the factor $\mathrm{e}^{\mathrm{j} \gamma(\xi)}$, the real part of the electric field and of the reduced electric field $y$-component radiated by an antenna along a line are reported in Figure 3. The considered antenna is a uniform planar circular array with radius equal to $20 \lambda$, lying in the plane $z=0$. Its elements, radially and azimuthally spaced of $0.8 \lambda$, are elementary Huygens sources linearly polarized along the $y$ axis. Accordingly, an oblate ellipsoidal modelling with $2 a=40 \lambda$ and $2 b=5 \lambda$ has been used. The straight line is the $x$-directed line at $y=0$, lying on a plane at distance $d=12 \lambda$ from the antenna. As can be seen, as $x$ increases, the spatial variations of the real part of the reduced field become increasingly slower than those of the electric field, since the local bandwidth has been minimized by choosing the optimal phase function. Quite analogous results are obtained for the imaginary parts.

To highlight the role of the optimal parameter $\xi$, the amplitude of the electric field $y$-component along the same

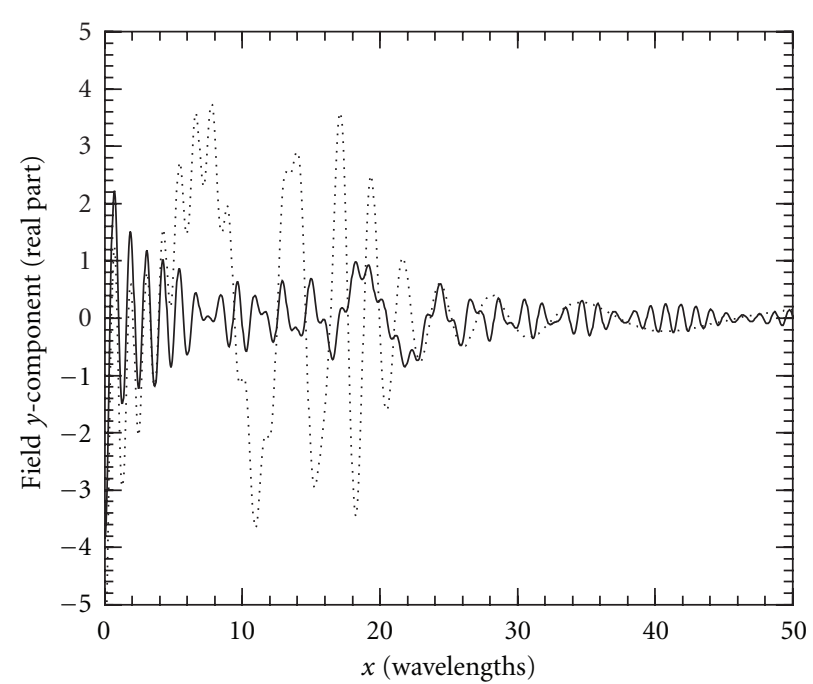

FIGURE 3: Real part of the NF $y$-component on the line $y=0, z=$

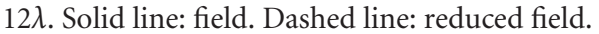

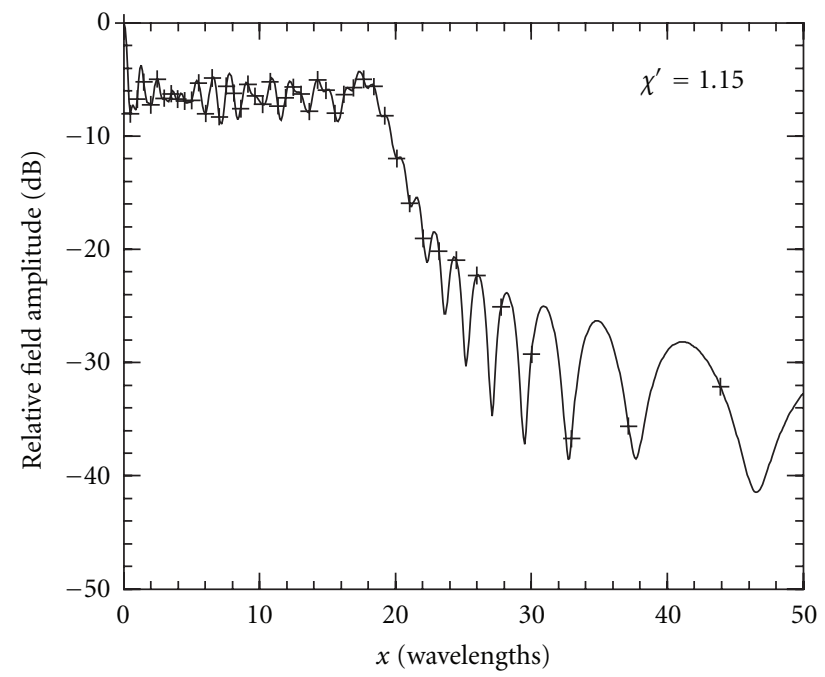

FIGURE 4: Amplitude of the NF $y$-component on the line $y=0, z$ $=12 \lambda$ as function of $x$. The samples distribution (crosses) when adopting a uniform sampling in $\xi$ is also shown.

line is plotted as function of $x$ and $\xi$ in Figures 4 and 5. As can be seen, when using $\xi$, the spatial variations are made uniform, by reducing the scale when these variations slow down. Thus, a uniform sampling in $\xi$ gives rise to a denser samples distribution where $w(\xi)$ is greater and to a sparser one in the zones characterized by smaller values of $w(\xi)$ (see Figure 4).

\section{Representation of the Probe Voltage on a Scanning Surface}

Let us consider an AUT enclosed in a convex domain bounded by a rotational surface $\Sigma$ and a nondirective probe scanning an arbitrary surface obtained by rotating a meridian curve always external to the cone of vertex at 
the observation point $P$ and tangent to $\Sigma$. Since the voltage $V$ measured by such a kind of probe has essentially the same effective spatial bandwidth of the AUT field [16], the nonredundant sampling representations of EM fields can be applied to it.

As a consequence, the reduced voltage $\tilde{V}(\xi)=V(\xi) \mathrm{e}^{\mathrm{j} \gamma(\xi)}$ at $P(\xi(\vartheta), \varphi)$ on the meridian curve fixed by $\varphi$ can be evaluated via the OSI expansion

$$
\tilde{V}(\xi(\vartheta), \varphi)=\sum_{m=m_{0}-q+1}^{m_{0}+q} \tilde{V}\left(\xi_{m}, \varphi\right) \Omega_{M}\left(\xi-\xi_{m}\right) D_{M^{\prime \prime}}\left(\xi-\xi_{m}\right),
$$

where $m_{0}=\operatorname{Int}[\xi / \Delta \xi]$ is the index of sample nearest (on the left) to $P, 2 q$ is the number of the retained intermediate samples $\tilde{V}\left(\xi_{m}, \varphi\right)$, that is, the reduced voltages at the intersection points between the sampling parallels and the considered meridian curve, and

$$
\begin{gathered}
\xi_{m}=m \Delta \xi=\frac{2 \pi m}{\left(2 M^{\prime \prime}+1\right)}, \\
M^{\prime \prime}=\operatorname{Int}\left(\chi M^{\prime}\right)+1, \quad M^{\prime}=\operatorname{Int}\left(\chi^{\prime} W_{\xi}\right)+1,
\end{gathered}
$$

$\chi^{\prime}$ being a factor, slightly larger than one, controlling the band-limitation error, $\chi$ an analogous one controlling the truncation error $[7,15]$, and $\operatorname{Int}(x)$ denoting the integer part of $x$. Moreover,

$$
\begin{gathered}
D_{M^{\prime \prime}}(\xi)=\frac{\sin \left[\left(2 M^{\prime \prime}+1\right) \xi / 2\right]}{\left(2 M^{\prime \prime}+1\right) \sin (\xi / 2)}, \\
\Omega_{M}(\xi)=\frac{T_{M}\left[2 \cos ^{2}(\xi / 2) / \cos ^{2}(\bar{\xi} / 2)-1\right]}{T_{M}\left[2 / \cos ^{2}(\bar{\xi} / 2)-1\right]},
\end{gathered}
$$

are the Dirichlet and Tschebyscheff Sampling functions, respectively, $T_{M}(\cdot)$ being the Tschebyscheff polynomial of degree $M=M^{\prime \prime}-M^{\prime}$ and $\bar{\xi}=q \Delta \xi$.

The intermediate samples $\widetilde{V}\left(\xi_{m}, \varphi\right)$ can be evaluated via the OSI formula

$$
\begin{aligned}
& \tilde{V}\left(\xi_{m}, \varphi\right) \\
& \quad=\sum_{n=n_{0}-p+1}^{n_{0}+p} \tilde{V}\left(\xi_{m}, \varphi_{n, m}\right) \Omega_{N_{m}}\left(\varphi-\varphi_{n, m}\right) D_{N_{m}^{\prime \prime}}\left(\varphi-\varphi_{n, m}\right),
\end{aligned}
$$

where $\tilde{V}\left(\xi_{m}, \varphi_{n, m}\right)$ are the reduced samples on the parallel fixed by $\xi_{m}, 2 p$ is the retained samples number, $n_{0}=$ $\operatorname{Int}\left(\varphi / \Delta \varphi_{m}\right)$, and

$$
\begin{gathered}
\varphi_{n, m}=n \Delta \varphi_{m}=\frac{2 \pi n}{2 N_{m}^{\prime \prime}+1}, \\
N_{m}^{\prime \prime}=\operatorname{Int}\left(\chi N_{m}^{\prime}\right)+1, \\
N_{m}^{\prime}=\operatorname{Int}\left[\chi^{*} W_{\varphi}\left(\xi_{m}\right)\right]+1, \\
N_{m}=N_{m}^{\prime \prime}-N_{m}^{\prime}, \\
\chi^{*}=1+\left(\chi^{\prime}-1\right)\left[\sin \vartheta\left(\xi_{m}\right)\right]^{-2 / 3} .
\end{gathered}
$$

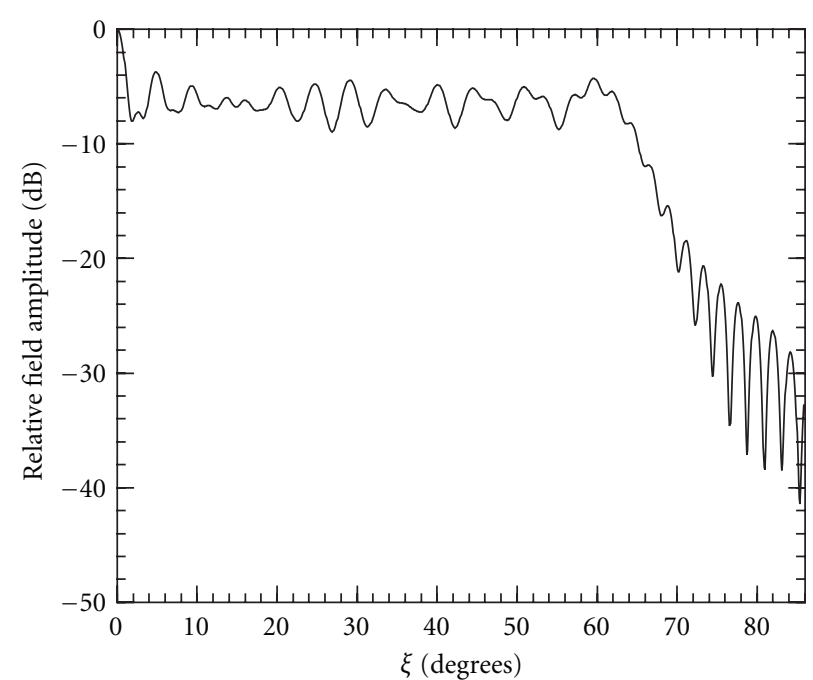

Figure 5: Amplitude of the NF $y$-component on the line $y=0, z=$ $12 \lambda$ as function of the optimal parameter $\xi$.

The variation of $\chi^{*}$ with $\xi$ is required to ensure a bandlimitation error constant with respect to $\xi[1]$.

As mentioned in the Introduction, above representations have been applied to a large variety of source and scanning geometries, showing that they are indeed robust against noise and that with $\chi^{\prime}=\chi \approx 1.2$ and $p=6-7$ we get absolute relative errors of the order of $-60 \mathrm{~dB}$ and a mean square one about $10 \mathrm{~dB}$ lower.

As an example of these performances, we report in the following some numerical and experimental results relative to the cylindrical scanning geometry. Experimental results relative to the plane-polar geometry can be found in [16].

We consider an elongated AUT and a scanning cylinder of radius $d$. An effective modelling for such an antenna is obtained by choosing the surface $\Sigma$ enclosing it coincident with the smallest prolate ellipsoid having major and minor semiaxes equal to $a$ and $b$ (see Figure 6). By properly employing the OSI expansions (Note that, to have an equal amount of truncation error at both the cylinder ends, the distribution of rings must be symmetrical with respect to $z=0$. Therefore, their position is fixed according to the sampling law $\xi_{m}=m \Delta \xi+\Delta \xi / 4$, instead of (16). Moreover, in (15), $\left.m_{0}=\operatorname{Int}[(\xi-\Delta \xi / 4) / \Delta \xi]\right)(15)$ and (19), it is so possible to recover the probe and rotated probe voltages $V$ and $V^{\prime}$ at the points needed to carry out the classical NF-FF transformation with cylindrical scanning [3].

The effectiveness and robustness of the so-developed nonredundant cylindrical NF-FF transformation have been assessed by many numerical tests. The reported simulations refer to a uniform planar array of $\lambda / 2$-spaced elementary Huygens sources, polarized along the $z$ axis, and covering an elliptical zone in the plane $y=0$, with major and minor semiaxes equal to $25 \lambda$ and $6 \lambda$. Accordingly, a prolate ellipsoidal modelling with $2 a=50 \lambda$ and $2 b=12 \lambda$ has been used. The radius $d$ of the scanning cylinder is equal to $12 \lambda$, and its height $h$ is $160 \lambda$. The working frequency is $10 \mathrm{GHz}$, and an open-ended WR-90 rectangular waveguide is chosen 


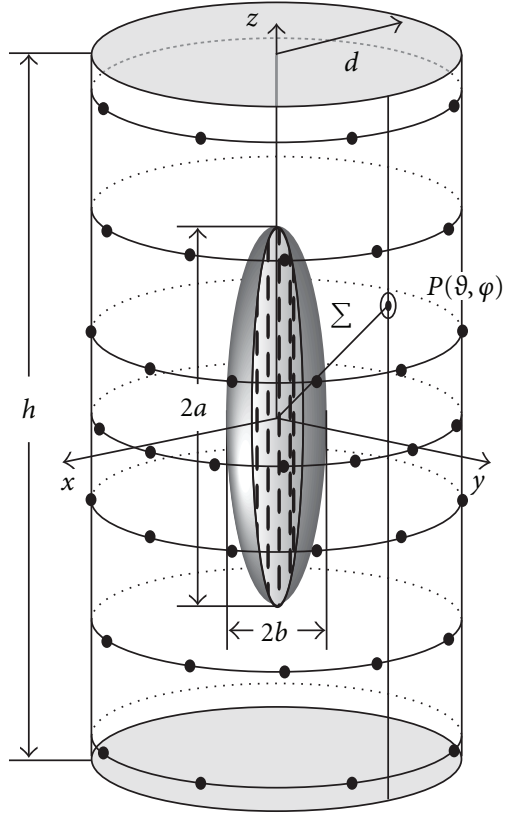

Figure 6: Cylindrical scanning: the prolate ellipsoidal modelling.

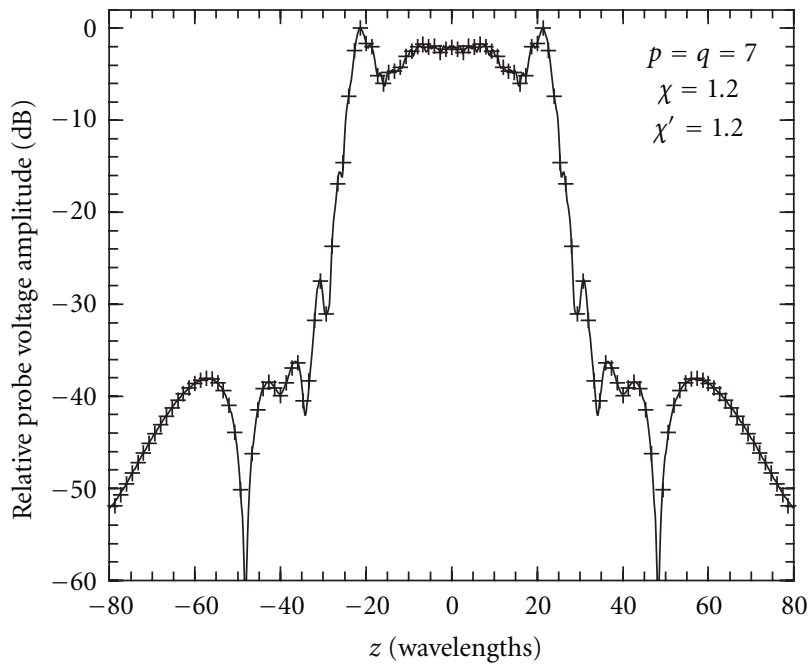

Figure 7: Amplitude of the probe output voltage $V$ on the generatrix at $\varphi=90^{\circ}$. Solid line: exact. Crosses: interpolated.

as probe. Figure 7 shows a representative reconstruction example of the amplitude of the voltage $V$ (the most significant one) over the cylinder generatrix at $\varphi=90^{\circ}$. As can be seen, there is an excellent agreement between the exact voltage and the reconstructed one. The accuracy in the NF interpolation is confirmed also by the maximum and meansquare error values. They are obtained by comparing the interpolated values of $V$ with those directly evaluated on a close grid in the central zone of the scanning cylinder, so that the existence of the guard samples is assured. Figure 8 shows the mean-square error, normalized to the voltage maximum value over the cylinder, for $p=q$ ranging from 3 to $12, \chi^{\prime}=1.20$, and $\chi=1.10,1.15,1.20$, and 1.25 . As

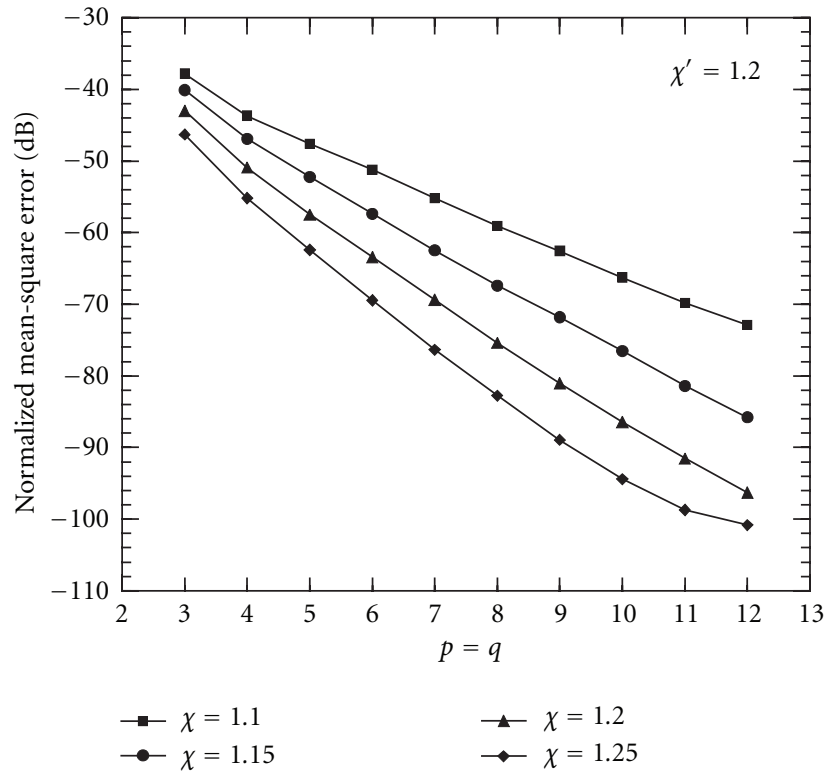

Figure 8: Mean-square reconstruction error of the probe voltage $V$.

expected, it decreases up to very low values on increasing the oversampling factor and/or the retained samples number. The algorithm stability has been investigated by adding random errors to the exact samples. These errors simulate a background noise (bounded to $\Delta a \mathrm{~dB}$ in amplitude and with arbitrary phase) and an uncertainty on the data of $\pm \Delta a_{r}$ $\mathrm{dB}$ in amplitude and $\pm \Delta \alpha$ degrees in phase. As shown in Figure 9, the algorithm is stable. The algorithm has been applied to recover the NF data needed for the classical probe compensated NF-FF transformation [3]. The reconstruction of the antenna FF pattern in the E-plane is reported in Figure 10. As can be seen, the exact and recovered fields are practically indistinguishable. It can be interesting to compare the number of the used NF samples (13566) with that (40 960) required by the classical NF-FF transformation [3].

The nonredundant NF-FF transformation with cylindrical scanning has been experimentally validated using the facilities available at the antenna characterization laboratory of the University of Salerno, where an advanced cylindrical NF measurement facility supplied by MI Technologies is available, and at the anechoic chamber of the University of Naples Parthenope, provided with a FF measurement range. The AUT, located in the plane $x=0$, is a very simple H-plane monopulse antenna, operating at $10 \mathrm{GHz}$ in the difference mode. It has been realized by using two pyramidal horns $(8.9 \times 6.8 \mathrm{~cm})$ of Lectronic Research Labs at a distance of $26 \mathrm{~cm}$ (between centers) and a hybrid Tee. The AUT has been modelled as enclosed in a prolate ellipsoid with major and minor semiaxes equal to $27 \mathrm{~cm}$ and $5 \mathrm{~cm}$. The probe voltages have been acquired on a cylinder with $d=16.6 \mathrm{~cm}$ and $h=240 \mathrm{~cm}$. To assess the effectiveness of the sampling representation, the amplitude and phase (in the range [$20 \mathrm{~cm}, 120 \mathrm{~cm}$ ], to improve its readability) of the reconstructed probe voltage relevant to the generatrix at $\varphi=0^{\circ}$ 


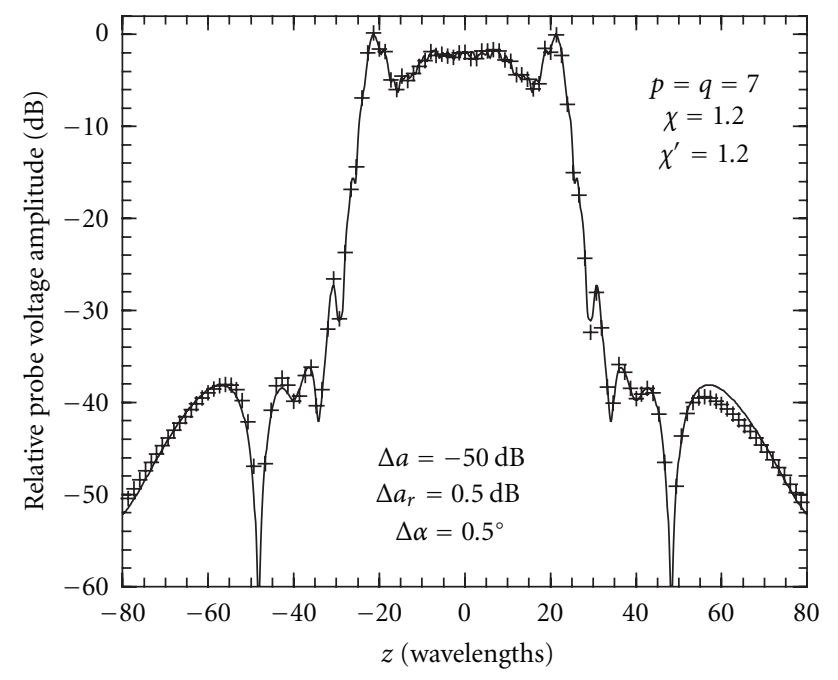

Figure 9: Amplitude of the probe output voltage $V$ on the generatrix at $\varphi=90^{\circ}$. Solid line: exact. Crosses: reconstructed from error affected data.

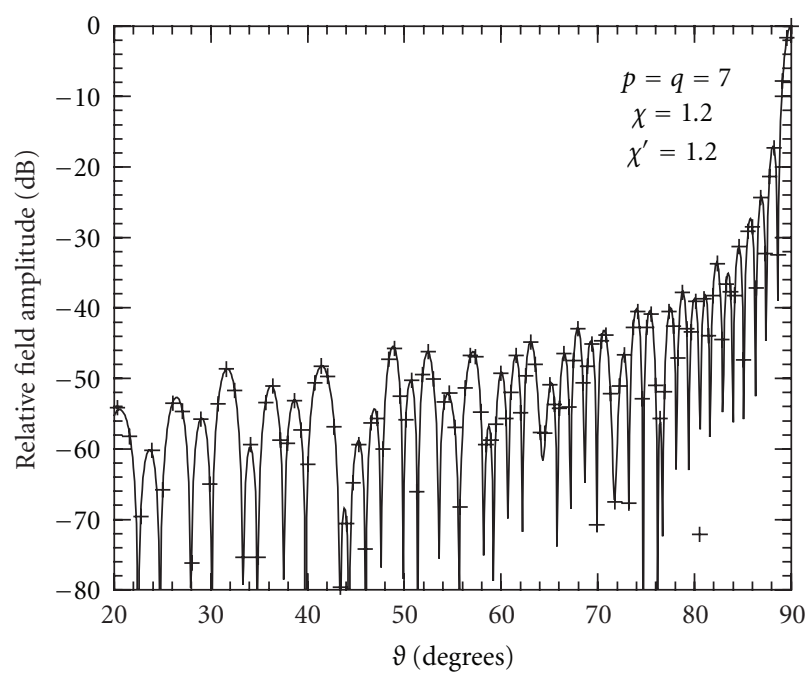

FIGURE 10: Far-field pattern in the E-plane. Solid line: exact field. Crosses: reconstructed from nonredundant cylindrical NF data.

are compared in Figures 11 and 12, respectively, with those directly measured on the same generatrix. As can be seen, the reconstruction is everywhere very good, save for the peripheral zone (below about $-60 \mathrm{~dB}$ ). The reconstructed voltage exhibits a smoother behaviour as compared to the measured one. This is due to the low pass filtering properties of the interpolation functions, which cut away the noise outside the AUT spatial bandwidth. All the reported reconstructions have been obtained by using $\chi^{\prime}=1.35$, $\chi=1.20$, and $p=q=8$. The comparison between the H-plane FF pattern reconstructed from the acquired nonredundant cylindrical NF data and that obtained directly from the data measured on the classical cylindrical grid (see Figure 13) assesses the overall effectiveness of the NF-FF transformation technique. In both cases, the software package MI-3000 has been used to get the FF reconstructions.

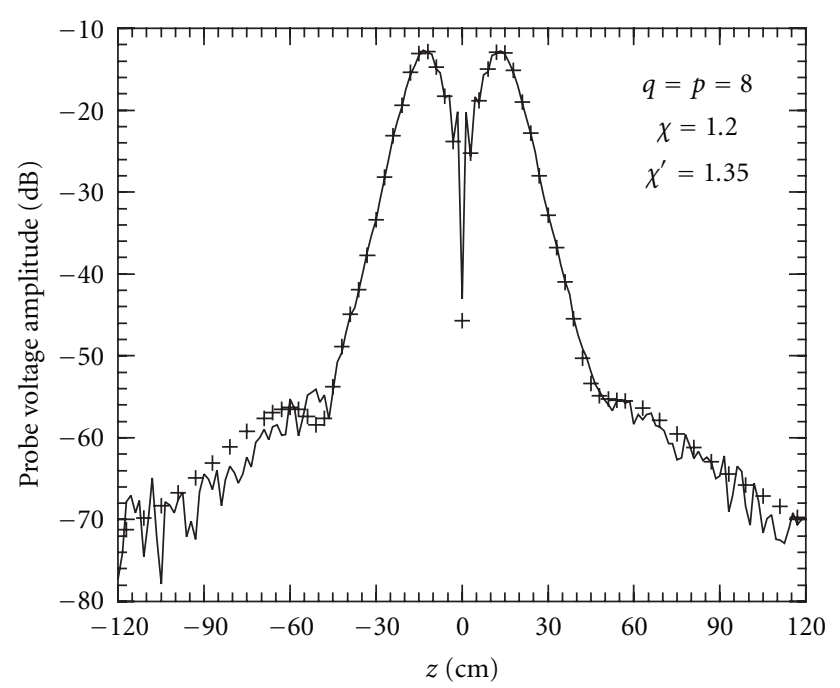

FIgURE 11: Amplitude of the probe output voltage $V$ on the generatrix at $\varphi=0^{\circ}$. Solid line: measured. Crosses: interpolated.

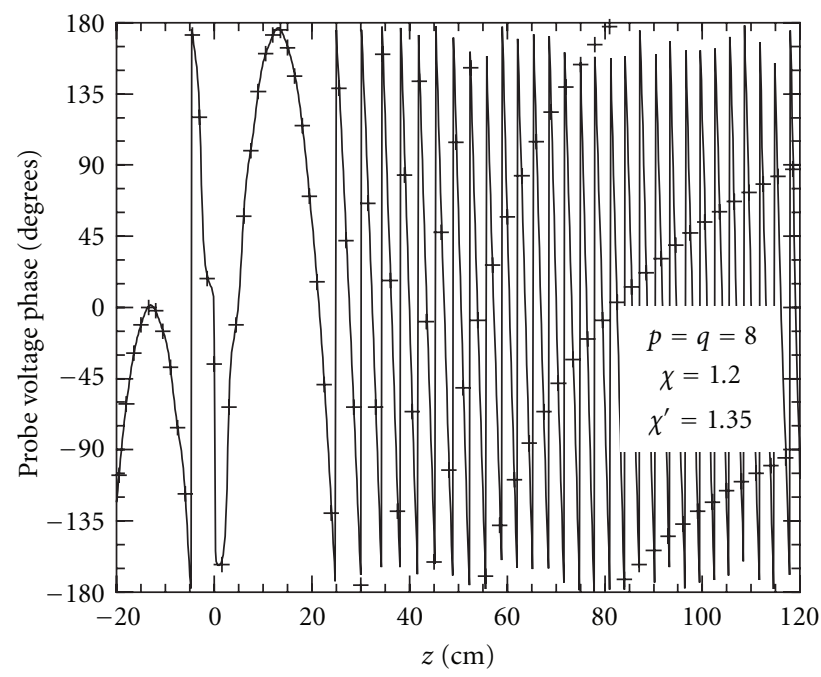

Figure 12: Phase of the probe output voltage $V$ on the generatrix at $\varphi=0^{\circ}$. Solid line: measured. Crosses: interpolated.

The accuracy of the FF reconstruction process is further confirmed by comparing (see Figure 14) the H-plane pattern obtained from the nonredundant measurements with that directly measured in the FF region at the anechoic chamber of the University of Naples Parthenope. As can be seen, although the measurements have been carried out with quite different techniques and environmental conditions, a very good agreement results, save for small discrepancies in the far-out side lobes region, due to the truncation of the scanning zone. It must be stressed that the described technique allows a significant reduction of the number of measurements, without losing the accuracy of the classical approach. As a matter of fact, the number of samples needed by the described NF-FF transformation with cylindrical scan is 1895 against that (5760) required by MI-3000 package to cover the same scanning zone. 


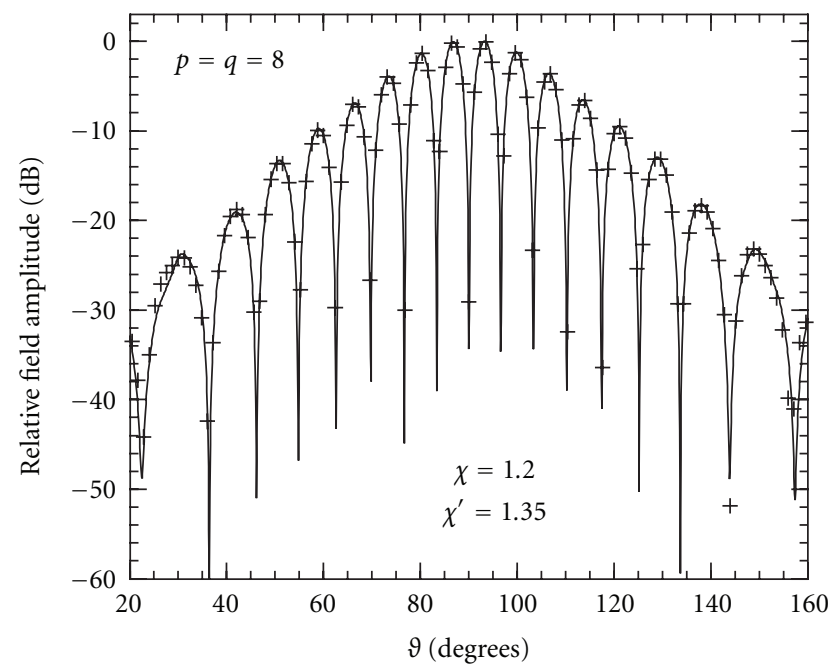

Figure 13: H-plane pattern. Solid line: obtained from NF data measured on the classical cylindrical grid. Crosses: reconstructed from NF data acquired via the nonredundant cylindrical scanning.

\section{Voltage Reconstruction from Nonredundant Samples along a Spiral}

As emphasized in the Introduction, the adoption of a spiral scanning can improve the performance of NF-FF measurement techniques by significantly reducing the measurement time. Accordingly, in this Section, we report the main results concerning the reconstruction of the probe voltage on a quite arbitrary rotational surface $\mathcal{M}$ (with the same rotational symmetry of the surface $\Sigma$ bounding the source) from the knowledge of a nonredundant number of its samples lying on a spiral wrapping the surface $[34,41]$.

In order to get such a sampling representation of the voltage, it is necessary [34]

(i) to choose the pitch of the spiral coincident with the sample spacing needed for the interpolation along a meridian curve,

(ii) to develop a nonredundant sampling representation of the voltage on the spiral, based on the theoretical results in [15].

As the sample spacing is uniform in the $\xi$ variable associated to $\Sigma$ (see formula (7)), condition (i) implies that the spiral equation is

$$
\xi=k \phi,
$$

where $\phi$ is the angular parameter describing the spiral.

Such a spiral can be viewed as intersection of the surface $\mathcal{M}$ with the line $\xi=$ const in the meridian plane, emanating from a point which moves on a spiral that wraps, with a constant pitch, the surface $\Sigma$ enclosing the AUT. As the variable $\xi$ varies in the range $[-\pi, \pi]$, the aforementioned condition on the spiral pitch implies that the constant $k$ must be such that the interval $\Delta \xi=2 \pi k$ between two consecutive intersections of the spiral with the meridian curve is equal

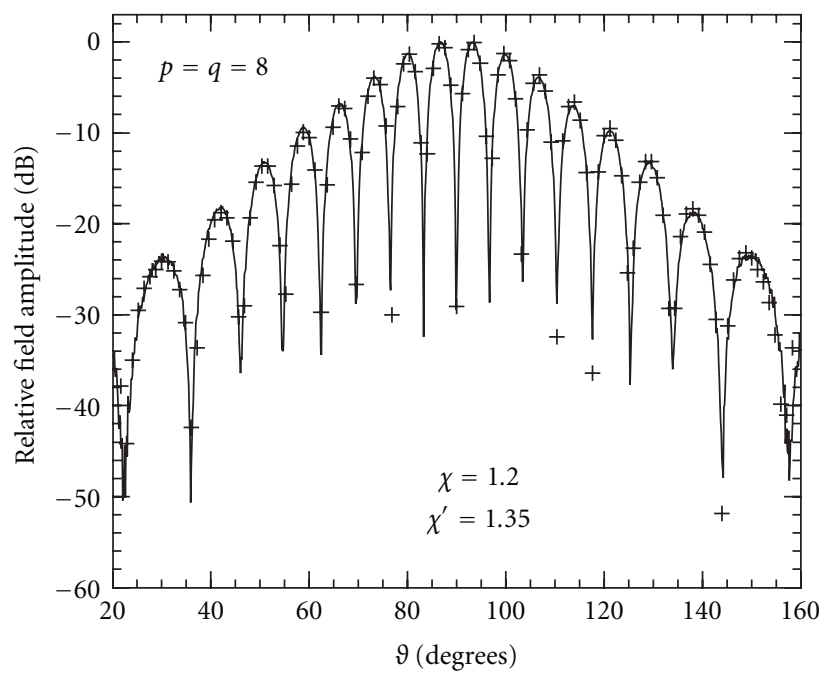

Figure 14: H-plane pattern. Solid line: direct FF measurements. Crosses: reconstructed from NF data acquired via the nonredundant cylindrical scanning.

to $2 \pi /\left(2 M^{\prime \prime}+1\right)$, with $M^{\prime \prime}=\operatorname{Int}\left(\chi M^{\prime}\right)+1$, and $M^{\prime}=$ $\operatorname{Int}\left(\chi^{\prime} W_{\xi}\right)+1$. It follows that $k=1 /\left(2 M^{\prime \prime}+1\right)$.

The determination of optimal phase factor $\psi$ and parameter $\eta$ to get a nonredundant representation of the voltage along the spiral is a more difficult task, as the influence of the source geometry is more involved. In the case of a spherical source, the result can be obtained [34] by observing that the extreme values of $\hat{R} \cdot \hat{t}$ in (3) and (4) are determined by considering the intersection of the plane defined by $\hat{t}$ and the unit vector $\hat{r}$ with the cone having the vertex at the generic point $Q$ on the spiral and the generatrices coincident with the tangents to the sphere modelling the AUT. Denoting by $\hat{R}_{1,2}$ the related unit vectors and by $\varepsilon$ the angle between $\hat{r}$ and $\hat{t}$ (Figure 15), we get [34] the following

$$
\begin{gathered}
\frac{\left(\hat{R}_{1}+\hat{R}_{2}\right)}{2}=\hat{r} \sin \delta=\hat{r} \sqrt{1-\frac{a^{2}}{r^{2}},} \\
\frac{\left(\hat{R}_{1}-\hat{R}_{2}\right) \cdot \hat{t}}{2}=\cos \delta \sin \varepsilon=\left(\frac{a}{r}\right) \sin \varepsilon .
\end{gathered}
$$

By substituting (22) in (3), and taking into account that $\mathrm{d} r=\hat{r} \cdot \hat{t} \mathrm{~d} s$, it results in the following:

$$
\begin{aligned}
\psi & =\beta \int_{0}^{r} \sqrt{1-\frac{a^{2}}{r^{2}}} \mathrm{~d} r \\
& =\beta \sqrt{r^{2}-a^{2}}-\beta a \cos ^{-1}\left(\frac{a}{r}\right) .
\end{aligned}
$$

On the other hand [34],

$$
\begin{gathered}
\mathrm{d} s=\sqrt{r^{2} \sin ^{2} \theta+k^{2} r^{2}+k^{2} \dot{r}^{2}} \mathrm{~d} \phi, \\
\sin \varepsilon=\sqrt{1-(\hat{r} \cdot \hat{t})^{2}},
\end{gathered}
$$




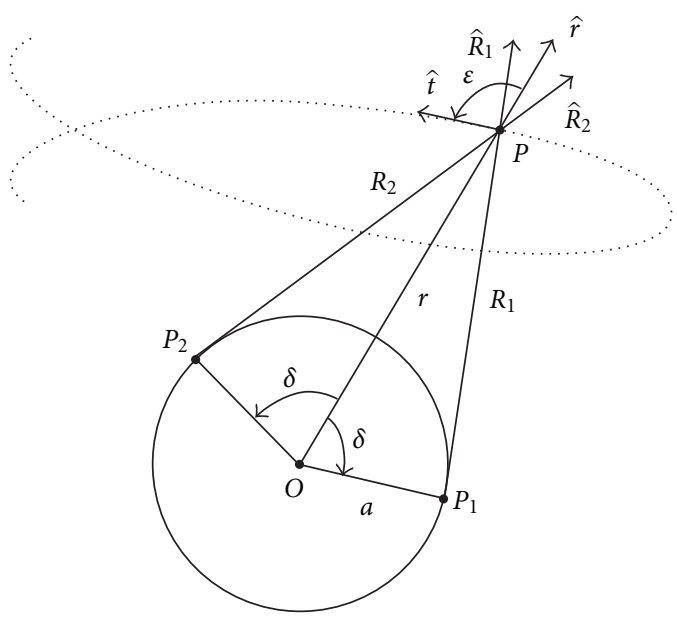

Figure 15: Geometry of the problem in the plane $\hat{t}, \hat{r}$.

wherein $\dot{r}=\mathrm{d} r / \mathrm{d} \vartheta$ and, being in such a case $\xi$ equal to the polar angle $\vartheta$,

$$
\begin{aligned}
\hat{r} \cdot \hat{t} & =\frac{\mathrm{d} r}{\mathrm{~d} s}=\frac{\mathrm{d} r}{\mathrm{~d} \phi} \frac{\mathrm{d} \phi}{\mathrm{d} s} \\
& =\left[\frac{\mathrm{d} r}{\mathrm{~d} \vartheta} \frac{\mathrm{d} \vartheta}{\mathrm{d} \phi}\right] \frac{\mathrm{d} \phi}{\mathrm{d} s} \\
& =k \dot{r} \frac{\mathrm{d} \phi}{\mathrm{d} s}=\frac{k \dot{r}}{\sqrt{r^{2} \sin ^{2} \vartheta+k^{2} r^{2}+k^{2} \dot{r}^{2}}} .
\end{aligned}
$$

By substituting relations (23) and (25) in (4), it results:

$$
\eta=\frac{\beta a}{W_{\eta}} \int_{0}^{\phi} \sqrt{k^{2}+\sin ^{2} k \phi} \mathrm{d} \phi .
$$

It is worth noting that the expression (24) of the phase function $\psi$ relevant to the sampling representation along the spiral coincides with that $\gamma$ relevant to the representation on a meridian curve [15].

According to (27), the parameter $\eta$ is $\beta / W_{\eta}$ times the arclength along the spiral which wraps the sphere modelling the AUT. Since such a spiral is a closed curve, it is convenient to choose the bandwidth $W_{\eta}$ such that $\eta$ covers a $2 \pi$ range when the whole projecting curve on the sphere is described. As a consequence,

$$
W_{\eta}=\frac{\beta a}{\pi} \int_{0}^{\left(2 M^{\prime \prime}+1\right) \pi} \sqrt{k^{2}+\sin ^{2} k \phi} d \phi
$$

namely, the bandwidth $W_{\eta}$ is $\beta / \pi$ times the length of the spiral that wraps from pole to pole the sphere modelling the AUT.

Let us now turn to the case of nonspherical modelling of the source. By following a heuristic reasoning, one can determine [41] the phase factor $\psi$ and parameterization $\eta$ also in this case. In particular, by generalizing the corresponding relations for the case of spherical modelling (see (24), (27), and (28)), $\psi$ coincides with the phase function $\gamma$ relevant to a meridian curve, $\eta$ is $\beta / W_{\eta}$ times the arc length of the projecting point that lies on the spiral wrapping the surface
$\Sigma$, and the bandwidth $W_{\eta}$ is $\beta / \pi$ times the length of the spiral wrapping $\sum$ from pole to pole. Namely, the spiral, $\psi$ and $\eta$ are such that they coincide with those relevant to the spherical modelling when the surface $\Sigma$ leads to a sphere.

According to the above results, the OSI formula for reconstructing the reduced voltage at any point $Q$ of the spiral is $[34,41]$

$$
\tilde{V}(\eta)=\sum_{n=n_{0}-p+1}^{n_{0}+p} \tilde{V}\left(\eta_{n}\right) \Omega_{N}\left(\eta-\eta_{n}\right) D_{N^{\prime \prime}}\left(\eta-\eta_{n}\right),
$$

where $n_{0}=\operatorname{Int}(\eta / \Delta \eta)$ is the index of the sample nearest (on the left) to the point $Q, 2 p$ the number of retained samples $\widetilde{V}\left(\eta_{n}\right)$, and

$$
\eta_{n}=n \Delta \eta=2 \pi n /\left(2 N^{\prime \prime}+1\right)
$$

with $N^{\prime \prime}=\operatorname{Int}\left(\chi N^{\prime}\right)+1$ and $N^{\prime}=\operatorname{Int}\left(\chi^{\prime} W_{\eta}\right)+1$.

It must be stressed that, when interpolating the voltage in the neighbourhood of the poles $(\vartheta=0$ and $\vartheta=\pi)$, the enlargement bandwidth factor $\chi^{\prime}$ must be properly increased to avoid a significant growth of the band-limitation error. This is due to the fact that small variations of $\eta$ correspond to very large changes of $\phi$ in these zones.

The OSI expansion (29) can be employed to evaluate the reduced voltage values at the intersection points between the spiral and the meridian curve passing through the observation point $P$. Once these intermediate samples have been determined, the reduced voltage at $P$ can be reconstructed via the following OSI formula:

$$
\tilde{V}(\xi(\vartheta), \varphi)=\sum_{m=m_{0}-q+1}^{m_{0}+q} \tilde{V}\left(\xi_{m}\right) \Omega_{M}\left(\xi-\xi_{m}\right) D_{M^{\prime \prime}}\left(\xi-\xi_{m}\right),
$$

wherein $m_{0}=\operatorname{Int}\left[\left(\xi-\xi_{0}\right) / \Delta \xi\right]$ is the index of sample nearest (on the left) to $P, \widetilde{V}\left(\xi_{m}\right)$ are the intermediate samples,

$$
\xi_{m}=\xi_{m}(\varphi)=k \varphi+m \Delta \xi=\xi_{0}+m \Delta \xi
$$

and the other symbols have the same meanings as in (15).

In order to illustrate above results and for its practical relevance, let us consider the case of an elongated AUT whose NF is probed on a proper helix lying on a cylinder of radius $d$ (Figure 16). For such a kind of antenna, a flexible modelling, which can be a convenient alternative to the prolate ellipsoidal one, is the rounded cylinder modelling. It is obtained by choosing $\Sigma$ coincident with a cylinder of height $h^{\prime}$ ended in two half-spheres of radius $a^{\prime}$ (Figure 16). By taking into account that in the helicoidal scanning case $r(\vartheta)=d / \sin \vartheta$ and imposing the passage of the helix through a fixed point $Q_{0}$ of the generatrix at $\varphi=0$, the helix equations read

$$
\begin{gathered}
x=d \cos \left(\phi-\phi_{s}\right), \\
y=d \sin \left(\phi-\phi_{s}\right), \\
z=d \cot [\vartheta(\xi)],
\end{gathered}
$$

wherein $\phi_{s}$ is the value of $\phi$ at $Q_{0}$. 


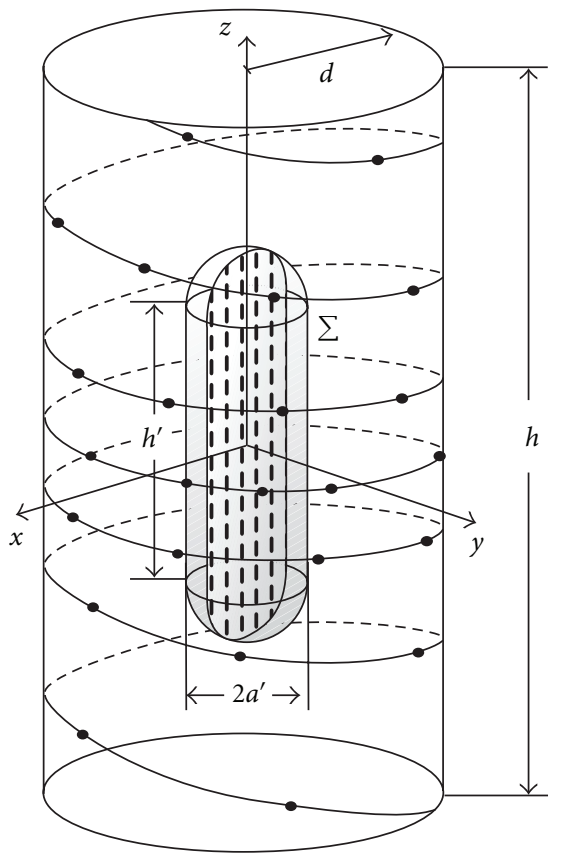

Figure 16: Helicoidal scanning: the rounded cylinder modelling.

It can be easily verified (see Figure 17) that, for the considered source modelling, the parameters involved in the expressions (6) and (7) of the phase factor and optimal parameter relevant to the nonredundant representation along a generatrix are $[22,36]$

$$
\begin{gathered}
\ell^{\prime}=2\left(h^{\prime}+\pi a^{\prime}\right), \\
R_{1,2}=\sqrt{\left(z \mp \frac{h^{\prime}}{2}\right)^{2}+d^{2}-a^{\prime 2},} \\
s_{1}^{\prime}=a^{\prime} \sin ^{-1}\left(\frac{a^{\prime} d+R_{1}\left(\left(\frac{h^{\prime}}{2}\right)-z\right)}{R_{1}^{2}+a^{\prime 2}}\right), \\
s_{2}^{\prime}=h^{\prime}+a^{\prime}\left[\pi-\sin ^{-1}\left(\frac{a^{\prime} d+R_{2}\left(\left(\frac{h^{\prime}}{2}\right)+z\right)}{R_{2}^{2}+a^{\prime 2}}\right)\right] .
\end{gathered}
$$

The reduced voltage at $P$ can be again reconstructed by means of (31), wherein the intermediate samples are still determined via (29), but, having imposed in such a case the passage of the helix through the point $Q_{0}$, the expressions of $\xi_{m}, \eta_{n}$, and $n_{0}$ become $\xi_{m}=\xi_{m}(\varphi)=\xi\left(\phi_{s}\right)+k \varphi+m \Delta \xi=$ $\xi_{0}+m \Delta \xi, n_{0}=\operatorname{Int}\left[\left(\eta-\eta_{0}\right) / \Delta \eta\right]$, and $\eta_{n}=\eta\left(\phi_{s}\right)+n \Delta \eta=$ $\eta_{0}+2 \pi n /\left(2 N^{\prime \prime}+1\right)$.

By properly employing the OSI expansions (29) and (31), it is so possible to recover the probe and rotated probe voltages $V$ and $V^{\prime}$ at the points needed to perform the classical NF-FF transformation with cylindrical scanning [3], thus getting a nonredundant NF-FF transformation with helicoidal scanning.

Such a NF-FF transformation has been numerically assessed in [36], obtaining results quite similar (in terms of accuracy and data reduction) to those reported in

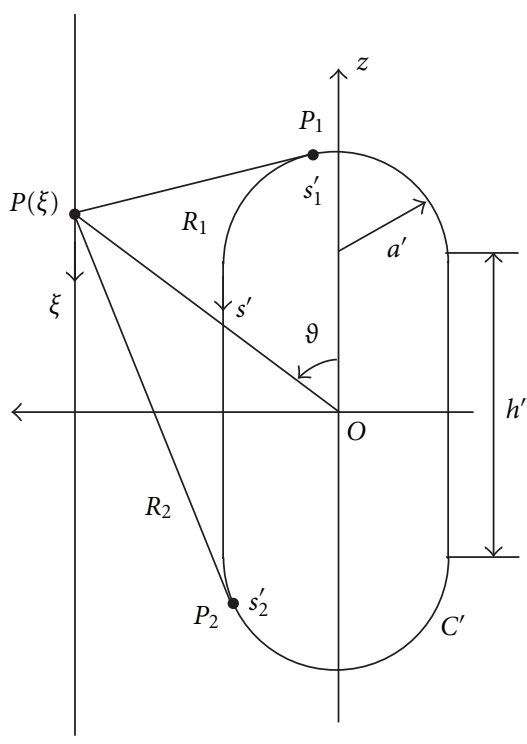

FIGURE 17: Relevant to a cylinder generatrix.

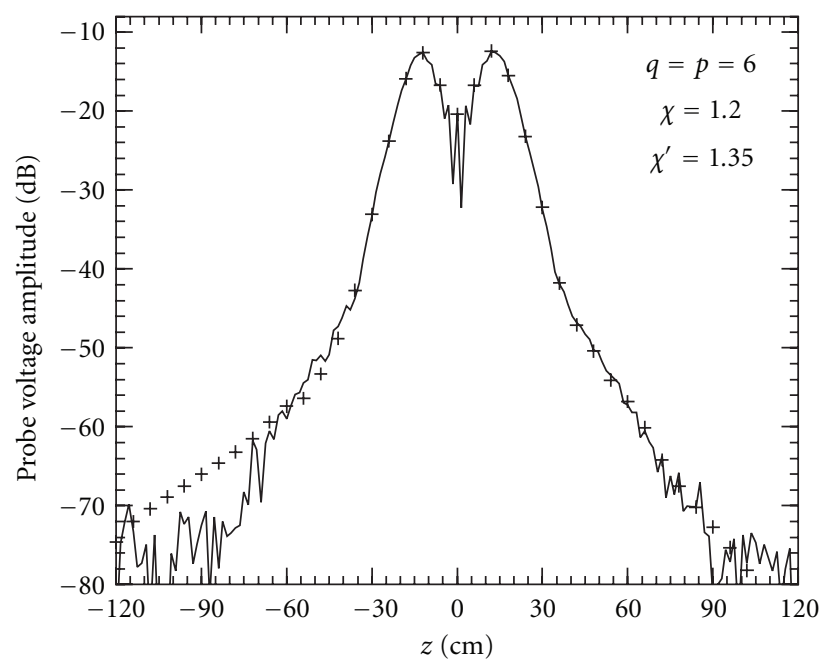

FIGURe 18: Amplitude of the probe output voltage $V$ on the generatrix at $\varphi=0^{\circ}$. Solid line: measured. Crosses: interpolated.

the previous section with reference to the nonredundant cylindrical NF-FF transformation.

Some experimental results performed at the antenna characterization laboratory of the University of Salerno and assessing the validity of the technique are reported in the following. The dimensions of the cylinder wrapped by the helix, the probe, the working frequency, and the AUT are the same as in Section 3. In such a case, the AUT operates in the sum mode and is modelled by a rounded cylinder with $h^{\prime}=35 \mathrm{~cm}$ and $a^{\prime}=3.4 \mathrm{~cm}$. In Figures 18 and 19, the amplitude and phase of the reconstructed probe voltage relevant to the generatrix at $\varphi=0^{\circ}$ are compared with those directly measured on the same generatrix. The same comments already done with reference to Figures 11 and 12 hold. The overall effectiveness of the described NF-FF transformation is assessed by comparing (Figures 20 and 21) the 


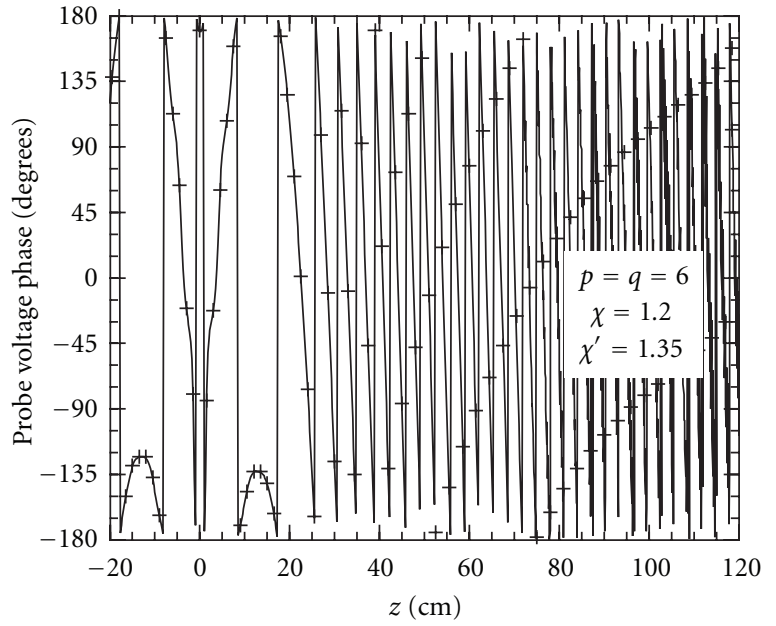

FIGURE 19: Phase of the probe output voltage $V$ on the generatrix at $\varphi=0^{\circ}$. Solid line: measured. Crosses: interpolated.

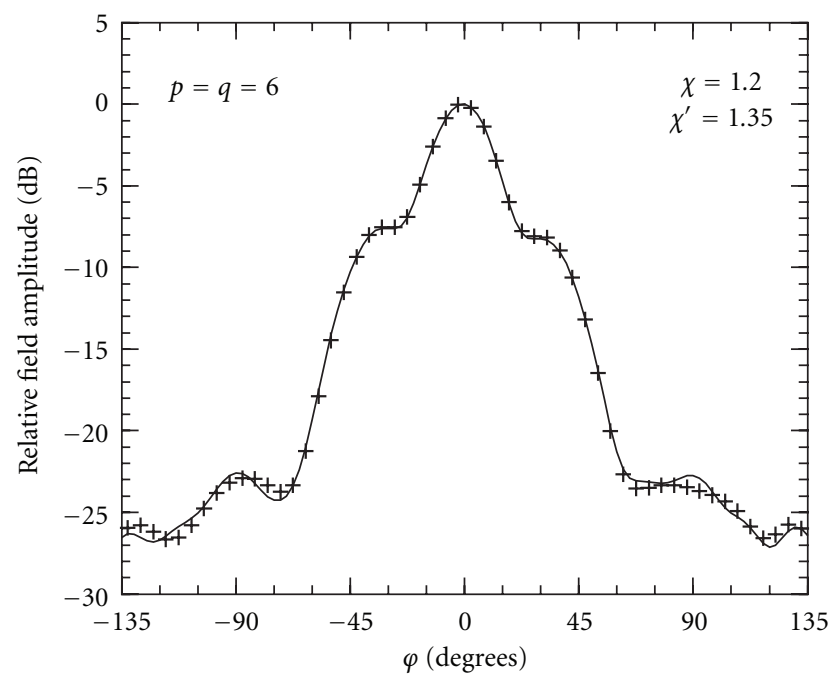

Figure 20: E-plane pattern. Solid line: reference. Crosses: reconstructed from NF data acquired via the helicoidal scanning.

FF patterns in the principal planes $\mathrm{E}$ and $\mathrm{H}$ reconstructed from the acquired NF data with those (references) obtained by using the software package MI-3000 from the data directly measured on the classical cylindrical grid. The same software has been used to get the FF reconstruction from the helicoidal NF data. To this end, the described OSI algorithm has been used for recovering the required cylindrical NF data. Moreover, the H-plane pattern obtained from the helicoidal measurements is compared in Figure 22 with that directly measured in the FF zone. As can be seen, in all the cases, there is a very good agreement, thus confirming the effectiveness of the technique.

It is interesting to compare the number of samples (948) needed by such a NF-FF transformation with helicoidal scan with that (5760) required by the traditional NF cylindrical scanning to cover the same measurement zone. As shown, the described technique allows a significant reduction of the

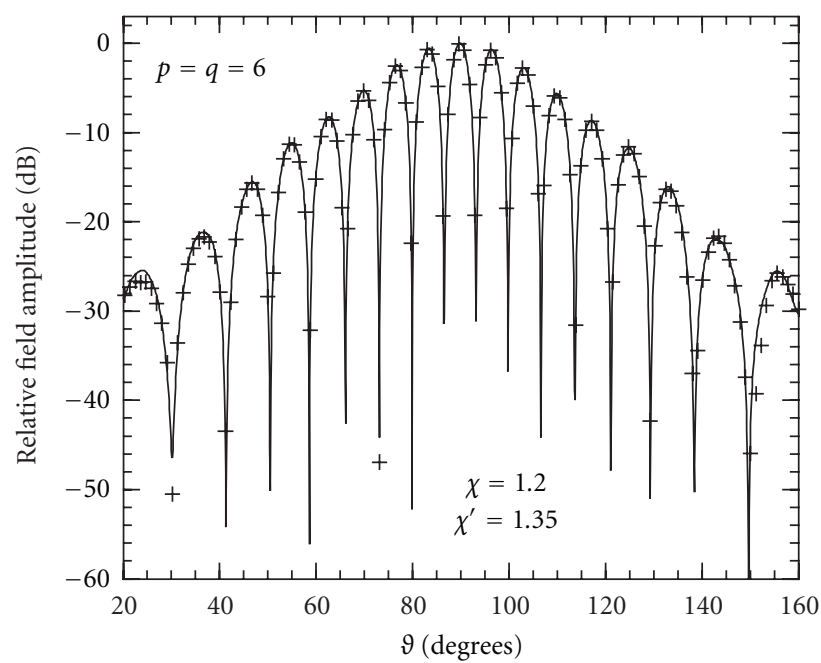

Figure 21: H-plane pattern. Solid line: reference. Crosses: reconstructed from NF data acquired via the helicoidal scanning.

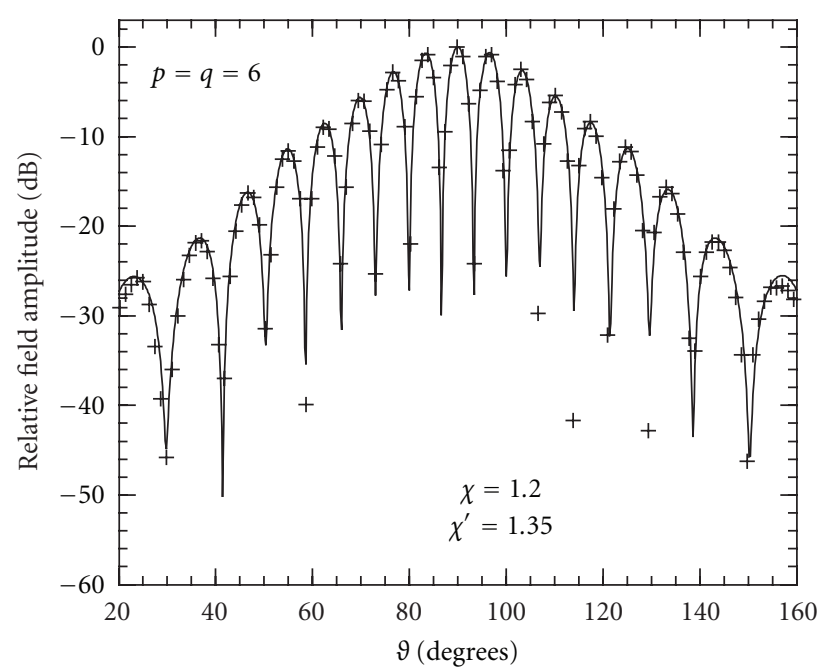

FIGURE 22: H-plane pattern. Solid line: direct FF measurements. Crosses: reconstructed from NF data acquired via the helicoidal scanning.

number of measurements, without losing the accuracy of the classical approach.

\section{Conclusions}

In this paper, we have presented an overview of the application of the band-limitation properties and nonredundant sampling representations of EM fields to NF-FF transformation techniques, outlining the remarkable reduction in the number of needed NF samples and measurement time so achievable in all conventional scannings. Then, the unified theory of the NF-FF transformations with spiral scannings has been reviewed highlighting that these transformations allow a further substantial reduction of the measurement time. In both cases, proper OSI formulas to efficiently 
reconstruct the NF data needed by the classical NF-FF transformation corresponding to the adopted scanning surface from the acquired nonredundant samples are reported. At last numerical, as well as, experimental results assessing the effectiveness of the considered NF-FF transformations are shown.

\section{References}

[1] O. M. Bucci and G. Franceschetti, "On the spatial bandwidth of scattered fields," IEEE Transactions on Antennas and Propagation, vol. 35, no. 12, pp. 1445-1455, 1987.

[2] O. M. Bucci and Gennarelli, "Use of sampling expansions in near-field-far-field transformation: the cylindrical case," IEEE Transactions on Antennas and Propagation, vol. 36, no. 6, pp. 830-835, 1988.

[3] W. M. Leach Jr. and D. T. Paris, "Probe compensated near-field measurements on a cylinder," IEEE Transactions on Antennas and Propagation, vol. 21, no. 4, pp. 435-445, 1973.

[4] A. D. Yaghjian, "Near-field antenna measurement on a cylindrical surface: a source scattering matrix formulation," NBS Technical Note 696, U.S. Government Printing Office, Washington, DC, USA, 1977.

[5] O. M. Bucci, G. D'Elia, and M. D. Migliore, “A new strategy to reduce the truncation error in near-field/far-field transformations," Radio Science, vol. 35, no. 1, pp. 3-17, 2000.

[6] O. M. Bucci and G. Di Massa, "Truncation error in the application of sampling series to electromagnetic problems," IEEE Transactions on Antennas and Propagation, vol. 36, no. 7, pp. 941-949, 1988.

[7] O. M. Bucci, C. Gennarelli, and C. Savarese, "Optimal interpolation of radiated fields over a sphere," IEEE Transactions on Antennas and Propagation, vol. 39, no. 11, pp. 1633-1643, 1991.

[8] O. M. Bucci, C. Gennarelli, and C. Savarese, "Fast and accurate near-field-far-field transformation by sampling interpolation of plane-polar measurements," IEEE Transactions on Antennas and Propagation, vol. 39, no. 1, pp. 48-55, 1991.

[9] L. I. Williams, Y. Rahmat-Samii, and R. G. Yaccarino, "The bipolar planar near-field measurement technique, part I: implementation and measurement comparisons," IEEE Transactions on Antennas and Propagation, vol. 42, no. 2, pp. 184-195, 1994.

[10] R. G. Yaccarino, Y. Rahmat-Samii, and L. I. Williams, "The bipolar planar near-field measurement technique, part II: nearfield to far-field transformation and holographic imaging methods," IEEE Transactions on Antennas and Propagation, vol. 42, no. 2, pp. 196-204, 1994.

[11] Y. Rahmat-Samii, L. I. Williams, and R. G. Yaccarino, "The UCLA bi-polar planar-near-field antenna-measurement and diagnostics range," IEEE Antennas and Propagation Magazine, vol. 37, no. 6, pp. 16-35, 1995.

[12] T. Brockett and Y. Rahmat-Samii, "A novel portable bipolar near-field measurement system for millimeter-wave antennas: construction, development, and verification," IEEE Antennas and Propagation Magazine, vol. 50, no. 5, pp. 121-130, 2008.

[13] O. M. Bucci and G. Franceschetti, "On the degrees of freedom of scattered fields," IEEE Transactions on Antennas and Propagation, vol. 37, no. 7, pp. 918-926, 1989.

[14] O. M. Bucci and T. Isernia, "Electromagnetic inverse scattering: retrievable information and measurement strategies," Radio Science, vol. 32, no. 6, pp. 2123-2137, 1997.

[15] O. M. Bucci, C. Gennarelli, and C. Savarese, "Representation of electromagnetic fields over arbitrary surfaces by a finite and nonredundant number of samples," IEEE Transactions on Antennas and Propagation, vol. 46, no. 3, pp. 351-359, 1998.

[16] O. M. Bucci, G. D’Elia, and M. D. Migliore, "Advanced field interpolation from plane-polar samples: experimental verification," IEEE Transactions on Antennas and Propagation, vol. 46, no. 2, pp. 204-210, 1998.

[17] O. M. Bucci, C. Gennarelli, G. Riccio, and C. Savarese, "Nearfield-far-field transformation from nonredundant plane-polar data: effective modellings of the source," IEE ProceedingsMicrowaves, Antennas and Propagation, vol. 145, no. 1, pp. 3338, 1998.

[18] O. M. Bucci, F. D’Agostino, C. Gennarelli, G. Riccio, and C. Savarese, "NF-FF transformation with plane-polar scanning: ellipsoidal modelling of the antenna," Automatika, vol. 41, no. 3-4, pp. 159-164, 2000.

[19] F. D’Agostino, C. Gennarelli, G. Riccio, and C. Savarese, "Data reduction in the NF-FF transformation with bi-polar scanning," Microwave and Optical Technology Letters, vol. 36, no. 1, pp. 32-36, 2003.

[20] F. Ferrara, C. Gennarelli, R. Guerriero, G. Riccio, and C. Savarese, "An efficient near-field to far-field transformation using the planar wide-mesh scanning," Journal of Electromagnetic Waves and Applications, vol. 21, no. 3, pp. 341-357, 2007.

[21] O. M. Bucci, C. Gennarelli, G. Riccio, C. Savarese, and V. Speranza, "Nonredundant representation of the electromagnetic fields over a cylinder with application to the near-field far-field transformation," Electromagnetics, vol. 16, no. 3, pp. 273-290, 1996.

[22] O. M. Bucci, C. Gennarelli, G. Riccio, and C. Savarese, "NFFF transformation with cylindrical scanning: an effective technique for elongated antennas," IEE Proceedings-Microwaves, Antennas and Propagation, vol. 145, no. 5, pp. 369-374, 1998.

[23] F. D'Agostino, F. Ferrara, C. Gennarelli, G. Riccio, and C. Savarese, "NF-FF transformation with cylindrical scanning from a minimum number of data," Microwave and Optical Technology Letters, vol. 35, no. 4, pp. 264-268, 2002.

[24] O. M. Bucci, F. D’Agostino, C. Gennarelli, G. Riccio, and C. Savarese, "Data reduction in the NF-FF transformation technique with spherical scanning," Journal of Electromagnetic Waves and Applications, vol. 15, no. 6, pp. 755-775, 2001.

[25] F. D’Agostino, F. Ferrara, C. Gennarelli, R. Guerriero, and M. Migliozzi, "Effective antenna modellings for NF-FF transformations with spherical scanning using the minimum number of data," International Journal of Antennas and Propagation, vol. 2011, Article ID 936781, 11 pages, 2011.

[26] J. C. Bolomey, B. J. Cown, G. Fine et al., "Rapid near-field antenna testing via arrays of modulated scattering probes," IEEE Transactions on Antennas and Propagation, vol. 36, no. 6, pp. 804-814, 1988.

[27] S. Pivnenko and T. Laitinen, "Spherical near-field antenna measurements with theta-scanning with reduced number of phi-steps," in Proceedings of the Antenna Measurement Techniques Association (AMTA '09), pp. 383-388, Salt Lake City, Utah, USA, November 2009.

[28] S. Pivnenko and T. Laitinen, "Fast pattern measurement of electrically large antennas by spherical near-field technique," in Proceedings of the 32nd ESA Workshop on Antennas for Space Applications, Noordwijk, the Netherlands, October 2010.

[29] Pivnenko S., "Time-saving scanning schemes for measurement of electrically large antennas by spherical near-field technique," in Proceedings of the European Conference on Antennas Propagation (EUCAP '11), pp. 2009-2013, Rome, Italy, April 2011. 
[30] R. G. Yaccarino, L. I. Williams, and Y. Rahmat-Samii, "Linear spiral sampling for the bipolar planar near-field antenna measurement technique," IEEE Transactions on Antennas and Propagation, vol. 44, no. 7, pp. 1049-1051, 1996.

[31] O. M. Bucci, C. Gennarelli, G. Riccio, and C. Savarese, "Nonredundant NF-FF transformation with helicoidal scanning," Journal of Electromagnetic Waves and Applications, vol. 15, no. 11, pp. 1507-1519, 2001.

[32] O. M. Bucci, F. D’Agostino, C. Gennarelli, G. Riccio, and C. Savarese, "Probe compensated far-field reconstruction by near-field planar spiral scanning," IEE Proceedings-Microwaves, Antennas and Propagation, vol. 149, no. 2, pp. 119-123, 2002.

[33] O. M. Bucci, F. D’Agostino, C. Gennarelli, G. Riccio, and C. Savarese, "Near-field-far-field transformation with spherical spiral scanning," IEEE Antennas and Wireless Propagation Letters, vol. 2, pp. 263-266, 2003.

[34] F. D’Agostino, C. Gennarelli, G. Riccio, and C. Savarese, "Theoretical foundations of near-field-far-field transformations with spiral scannings," Progress in Electromagnetics Research, vol. 61, pp. 193-214, 2006.

[35] F. D'Agostino, F. Ferrara, C. Gennarelli, R. Guerriero, and M. Migliozzi, "Near-field-far-field transformation technique with helicoidal scanning for elongated antennas," Progress in Electromagnetics Research B, vol. 4, pp. 249-261, 2008.

[36] F. D’Agostino, F. Ferrara, C. Gennarelli, R. Guerriero, M. Migliozzi, and G. Riccio, "NF-FF transformation with helicoidal scanning: an effective source modelling for elongated antennas," International Journal of Microwave and Optical Technology, vol. 3, no. 5, pp. 275-282, 2008.

[37] F. D’Agostino, F. Ferrara, J. A. Fordham et al., “An effective near-field-far-field transformation technique for elongated antennas using a fast helicoidal scan," IEEE Antennas and Propagation Magazine, vol. 51, no. 4, pp. 134-141, 2009.

[38] F. D’Agostino, F. Ferrara, C. Gennarelli, R. Guerriero, and M. Migliozzi, "Laboratory tests assessing the effectiveness of the NF-FF transformation with helicoidal scanning for electrically long antennaS," Progress in Electromagnetics Research, vol. 98, pp. 375-388, 2009.

[39] F. D’Agostino, F. Ferrara, C. Gennarelli, R. Guerriero, and M. Migliozzi, "An effective NF-FF transformation technique with planar spiral scanning tailored for quasi-planar antennas," IEEE Transactions on Antennas and Propagation, vol. 56, no. 9, pp. 2981-2987, 2008.

[40] F. D’Agostino, F. Ferrara, C. Gennarelli, R. Guerriero, M. Migliozzi, and G. Riccio, "A nonredundant near-field to farfield transformation with spherical spiral scanning for nonspherical antennas," The Open Electrical \& Electronic Engineering Journal, vol. 3, pp. 1-8, 2009.

[41] F. D’Agostino, F. Ferrara, C. Gennarelli, R. Guerriero, and M. Migliozzi, "The unified theory of near-field-far-field transformations with spiral scannings for nonspherical antennas," Progress In Electromagnetics Research B, no. 14, pp. 449-477, 2009.

[42] A. Capozzoli, C. Curcio, G. D’Elia, and A. Liseno, "Phaseless antenna characterization by effective aperture field and data representations," IEEE Transactions on Antennas and Propagation, vol. 57, no. 1, pp. 215-230, 2009.

[43] A. Capozzoli, C. Curcio, G. D’Elia, and A. Liseno, "SingularValue optimization in plane-polar near-field antenna characterization," IEEE Antennas and Propagation Magazine, vol. 52, no. 2, pp. 103-112, 2010.

[44] A. Capozzoli, C. Curcio, G. D'Elia et al., "A SVD-based approach to helicoidal NF-FF transformations," in Proceedings of the European Conference on Antennas Propagation (EUCAP '10), pp. 1-5, Barcelona, Spain, April 2010.

[45] S. Costanzo, G. Di Massa, and M. D. Migliore, "Integrated microstrip probe for phaseless near-field measurements on plane-polar geometry," Electronics Letters, vol. 37, no. 16, pp. 1018-1020, 2001.

[46] R. G. Yaccarino and Y. Rahmat-Samii, "Phaseless bi-polar planar near-field measurements and diagnostics of array antennas," IEEE Transactions on Antennas and Propagation, vol. 47, no. 3, pp. 574-583, 1999.

[47] S. F. Razavi and Y. Rahmat-Samii, "A new look at phaseless planar near-field measurements: limitations, simulations, measurements, and a hybrid solution," IEEE Antennas and Propagation Magazine, vol. 49, no. 2, pp. 170-178, 2007.

[48] S. Costanzo, G. Di Massa, and M. D. Migliore, "A novel hybrid approach for far-field characterization from nearfield amplitude-only measurements on arbitrary scanning surfaces," IEEE Transactions on Antennas and Propagation, vol. 53, no. 6, pp. 1866-1874, 2005. 

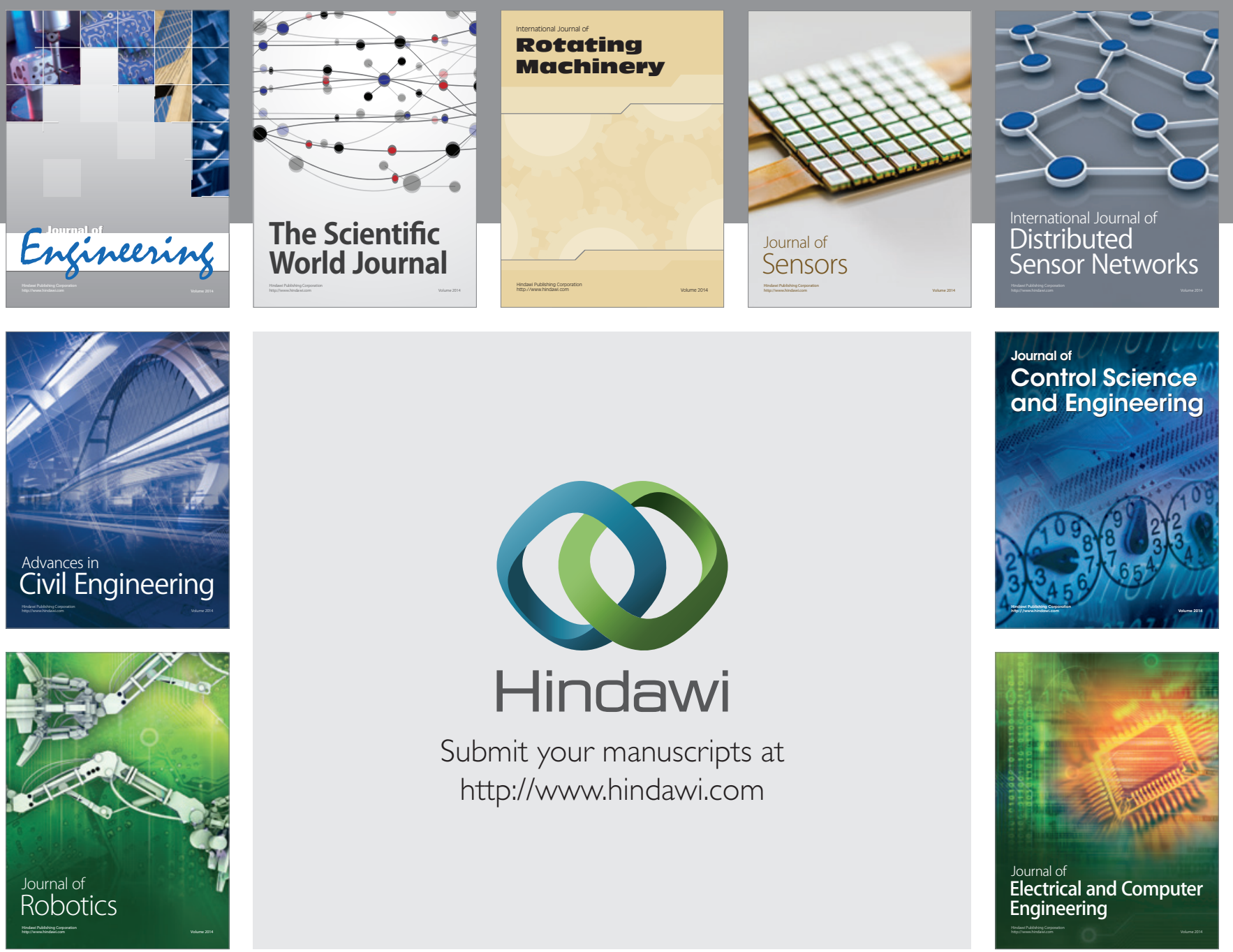

Submit your manuscripts at

http://www.hindawi.com
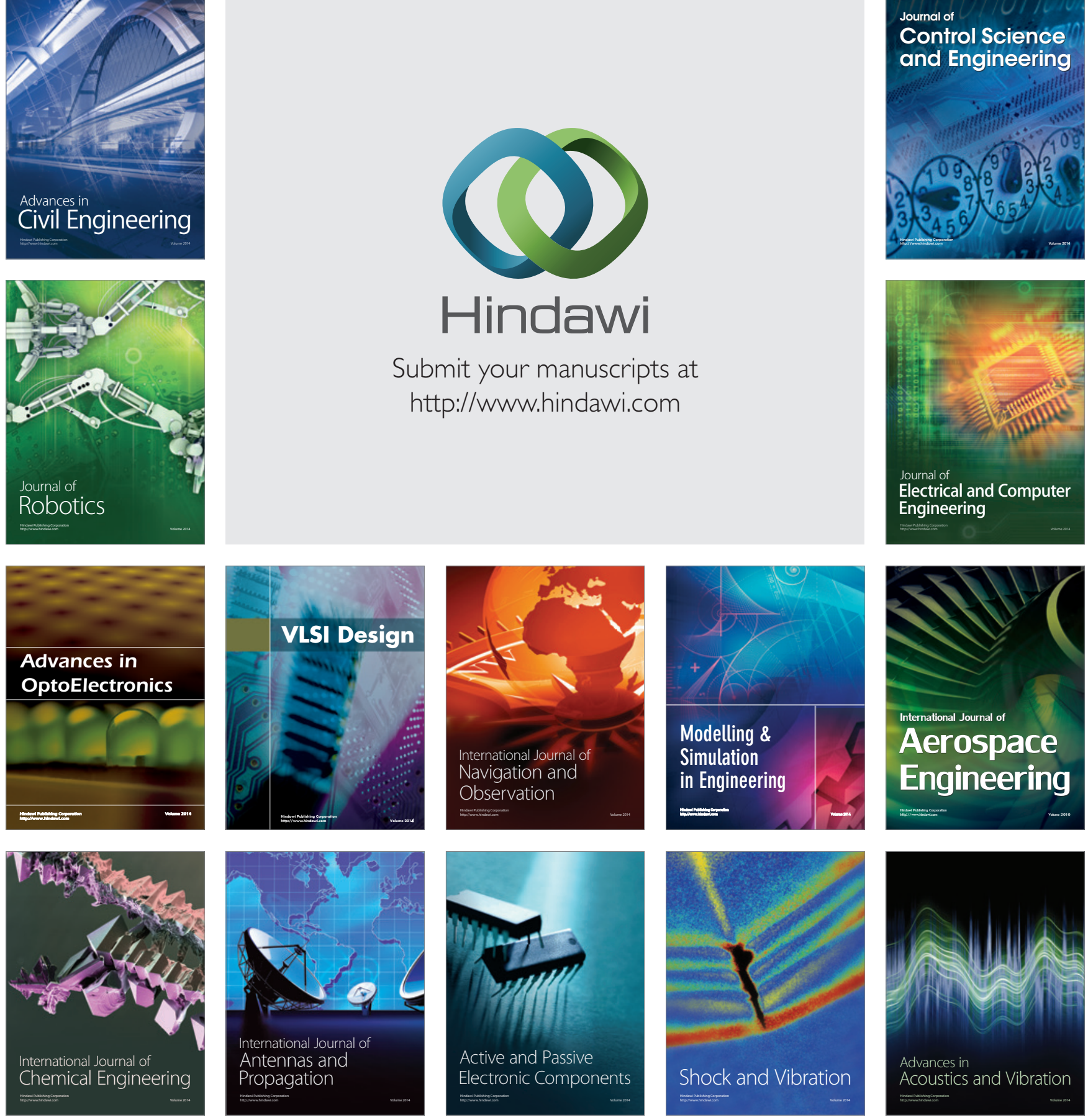